\title{
A new three-dimensional energy balance model for complex plant canopy geometries: Model development and improved validation strategies
}

\author{
Brian N. Bailey ${ }^{\mathrm{a}}$, Rob Stoll ${ }^{\mathrm{a}, *}$, Eric R. Pardyjak ${ }^{\mathrm{a}}$, Nathan E. Miller ${ }^{\mathrm{a}}$ \\ ${ }^{a}$ Department of Mechanical Engineering, University of Utah, Salt Lake City, UT, USA
}

\begin{abstract}
Canopy microclimate is a critical component of most biophysical processes in plants. Understanding the role of microclimate across scales in canopies with complex, heterogeneous architectures is challenging, as it is difficult to represent the relevant range of scales. In this study, a model was developed and validated to accurately predict the three-dimensional distribution of microclimate-related quantities (e.g., net radiation, surface temperature, evapotranspiration, flux partitioning) in complex canopy geometries. The modeling strategy was to aggregate individual leaves into isothermal sub-volumes, as resolving all leaves over whole-canopy scales is unfeasible. The model takes leaf-level models for convection, evapotranspiration, and radiative absorption/emission and integrates them over a discrete volume using the leaf angle probability distribution function. The model is built on a framework designed to utilize graphics processing units (GPUs) in order to offset the expense associated with model complexity, which can allow for the simulation of canopy-scale problems at sub-tree resolution. Additional cost-saving strategies are also suggested. Three-dimensional canopy energy transfer models have traditionally been difficult to validate. Two validation experiments were designed to simultaneously measure virtually all model outputs, often using multiple methods at many spatial locations. The model was able to reproduce point, 3D distributed, and bulk measurements at high accuracy, with average model errors within expected measurement errors.
\end{abstract}

Keywords: Canopy microclimate, Canopy radiation, Heterogeneous canopies, Leaf energy balance, Plant thermal imaging

\section{Introduction}

Canopy microclimate plays a role in nearly all biophysical processes in plants including respiration, photosynthesis, and growth (Jones, 2014). The temperature of individual plants in the canopy also affects the microclimate of organisms

\footnotetext{
* Corresponding author

Email address: rstoll@eng.utah.edu (Rob Stoll)
}

Preprint submitted to Agricultural and Forest Meteorology

November 19, 2015

(C) 2015. This manuscript version is made available under the Elsevier user license http://www.elsevier.com/open-access/userlicense/1.0/ 
living on or within plant tissues. These processes can have high sensitivity to leaf temperature, as temperature differences of only a degree can have critical implications on physiological function (Jones, 2004).

Leaf temperature can vary dramatically over short spatial distances due to step changes in radiation interception. In dense, closed canopies, radiative fluxes are relatively homogeneous in horizontal directions, which results in average temperature distributions that are primarily one-dimensional (Monteith and Unsworth, 2008). Many canopy energy models have been successfully developed which treat the canopy as a single 'big leaf' (e.g., Sinclair et al., 1976; Sellers et al., 1996), or several layers of big leaves (e.g., Leuning et al., 1995; Dai et al., 2004). However, in heterogeneous landscapes (e.g., urban canopies, savannas), temperature can vary in the horizontal direction by $10^{\circ} \mathrm{C}$ or more within a single tree crown (Tyree and Wilmot, 1990). This necessitates a fully threedimensional (3D) description of leaf temperature in these types of canopies.

Modeling 3D canopy microclimate presents several challenges, the foremost of which is accurately predicting net radiation fluxes, as radiation drives leaf temperature. As a result, leaf temperature is very sensitive to errors in predicted net radiation. During the day, radiative fluxes are usually very large, and dominate the leaf energy balance. At night, when net radiative fluxes are small, errors in radiation fluxes of only tens of $\mathrm{W} \mathrm{m}^{-2}$ can cause errors in leaf temperatures of several degrees or more.

Numerous models have been developed by past workers to simulate 3D radiation and temperature distributions in complex plant canopy geometries (Wang and Jarvis, 1990; Dauzat et al., 2001; Sinoquet et al., 2001; Luquet et al., 2003). These models have several limitations that reduce their utility. Radiation transfer is usually highly simplified in these models, with many important processes neglected such as scattering, anisotropic leaf inclination effects, and anisotropic emission. Radiation calculations are also typically very computationally expensive in complex canopies, which usually means that model domain sizes are limited to tree-scales rather than whole-canopy-scales (Bailey et al., 2014). This severely limits the range of length scales that can be modeled and makes it impossible to determine the effects of local heterogeneity on the larger plant community. Finally, 3D canopy microclimate models are traditionally poorly validated, as it is difficult to accurately measure 3D leaf temperature distributions (Ehleringer, 2000).

This study attempts to overcome these limitations by presenting a new scalable 3D energy transfer model for use in complex plant canopy geometries. The goal of the model is to accurately simulate the $3 \mathrm{D}$ distribution of microclimaterelated processes such as radiation transport, evapotranspiration (ET), heat flux partitioning, and leaf temperature over whole-canopy scales. These model outputs are critical inputs for models of biophysical processes such as photosynthesis and growth. Such a modeling system allows for direct scaling from leaves to canopies without assumptions of local homogeneity. Since an accurate and efficient radiation sub-model is a critical component of accomplishing this goal, the radiation transport model developed by Bailey et al. (2014) was used. This model includes radiation physics typically neglected in other models such 
as anisotropic scattering and emission. Computational expense is afforded by performing calculations in parallel on graphics processing units (GPUs), allowing the simulation of canopy-scale problems. Two experiments were conducted that were specially designed to provide a data set against which the present model could be rigorously evaluated.

\section{Model description}

The ultimate goal of the proposed modeling system is to simulate the $3 \mathrm{D}$ distribution of leaf surface temperature, as well as all contributing heat fluxes. This requires numerous coupled sub-models that provide the necessary information for the calculation of leaf temperature. An overview of the model flow is depicted in Fig. 1. In order for the model to calculate leaf surface temperature, it must also use sub-models to calculate various radiative fluxes, ground temperature, and latent and sensible heat fluxes from the leaf. Many of these sub-models are coupled, and thus require iteration. The following sections describe how the domain is constructed, and sub-model formulations.

\subsection{Numerical representation of the physical domain}

Given present computational capabilities, it is unreasonable to attempt to represent all individual leaves in an entire canopy, as this likely amounts to billions of leaves. To describe the local variability within the computational domain, objects (namely trees and the ground surface) are discretized into isothermal sections with constant properties as depicted in Fig. 2. The ground surface is discretized into sub-areas called patches. The tree crown is discretized into sub-volumes that aggregate the effects of individual leaves into homogeneous media. The shapes of the patches and volumes are arbitrary as long as they sufficiently describe spatial variation of properties (e.g., temperature, radiation, boundary-layer conductance). For the purposes of this study, the energy balance of woody material is not considered.

Vegetation within volumes are represented statistically by specifying the volumes leaf area density $a$, and a probability density function of leaf orientation $g_{L}$ (see Bailey et al., 2014). The average leaf reflectivity, transmissivity, and emissivity must be specified for all simulated wavelength bands of radiation. Finally, the index of refraction of leaf cuticle wax must also be specified for all simulated wavelength bands of radiation.

\subsection{Radiation transport model}

The radiation sub-model is described in full detail in Bailey et al. (2014), and only a brief overview is reproduced here. The radiation model uses a threedimensional deterministic ray tracing approach. The model is driven by user specification of the incoming solar radiation flux, the fraction of direct to diffuse solar radiation, and the incoming longwave radiation flux from the sky, which could come either from data or a sub-model. The model used here also approximates the solar radiation spectrum by partitioning it into a photosynthetically 
active or 'PAR' band (wavelengths less than $660 \mathrm{~nm}$ ) and a near infrared or 'NIR' band (wavelengths greater than $660 \mathrm{~nm}$ ). Note that these bands are not limitations of the model, as the solar radiation spectrum could be separated into an arbitrary number of bands.

As described above, individual leaves are not resolved, rather they are aggregated to form a continuous medium that is represented statistically. Simulated packets of photons or 'rays' are launched to and from ground patches to simulate the various modes of radiation transport in the natural environment. In the event that a ray intersects a vegetation volume, some fraction of that ray's energy is absorbed, scattered, or transmitted. These fractions are calculated according to anisotropic attenuation coefficients and scattering phase functions calculated for each volume using $g_{L}$. Scattered radiation is explicitly traced as it leaves the volume.

Model outputs are three-dimensional predictions of direct PAR, direct NIR, diffuse PAR, diffuse NIR, diffuse longwave, and emitted longwave radiative fluxes for each leaf volume and ground patch.

\subsubsection{Sampling errors}

Ray-tracing-based methods use a discrete number of rays to simulate the paths of a nearly continuous distribution of photons. As a result, a choice must be made regarding the number of discrete ray paths to trace. If not enough ray paths are used, undersampling can occur, which means that the ray discretization is not fine enough to adequately sample the domain space. This is a well-known problem in the field of computer graphics, which can result pixelated or otherwise unrealistic images (Cook, 1986). This phenomenon is commonly termed aliasing. In the context of the present model, undersampling can result in simulated radiation fields that do not vary smoothly in space, but rather they contain errors randomly distributed in space.

Sampling errors had the potential to become problematic in the present model, particularly for certain individual leaves or as vegetation volumes became small. These errors arise when there are not enough rays intersecting a given leaf or vegetation volume (undersampling). They can also occur when too many rays hit a given leaf or vegetation volume due to random errors (oversampling), although this type of sampling error was usually not problematic. Most often, the net radiation flux was under predicted as a result of sampling errors. This is because radiative losses (which are negative) are fixed, and not a function of the number of ray trajectories. However, undersampling can result in under prediction of the incoming radiative flux (positive), resulting in an overall under prediction of the net radiative flux.

Ideally, a very large number of ray trajectories should be simulated such that sampling errors tend toward zero. However, this can be very computationally expensive. Errors in the net radiative flux on the order of $10 \mathrm{~W} \mathrm{~m}^{-2}$ for any given leaf or volume may be acceptable in cases where, say, only the net radiative flux is of interest. However, we found that small sampling errors in the net radiative flux could translate into large local errors in temperature, particularly at night or for well-shaded leaves/volumes. 
Sampling errors were minimized using two techniques. The first was to add a stochastic component to the ray launching positions within patches and volumes, as is commonly done in Monte Carlo ray tracing (Mahan, 2002). Rather than launching rays from the center of patches and volumes for longwave calculations as in Bailey et al. (2014), rays were given a random initial position within patches or volumes. Note that incoming shortwave launches were still performed in a gridded pattern (cf. Bailey et al., 2014), as this was found to better reduce sampling errors than a stochastic formulation.

The second method utilized a radiative equilibrium test to correct for sampling errors in longwave calculations that could potentially result in a violation of the second law of thermodynamics. In a preprocessor step, an arbitrary constant temperature was chosen (e.g., $T=300 \mathrm{~K}$ ). All temperatures within the domain were fixed at this temperature $\left(T_{L}=T\right.$ and $\left.T_{G}=T\right)$. The incoming longwave flux from the sky was set to $\sigma T^{4}$, and all shortwave fluxes were set to zero. According to the second law of thermodynamics, the net radiative flux at all patches, leaves, and volumes should theoretically be zero in this case. Any deviation from zero was considered sampling error and a violation of the second law of thermodynamics. As the number of ray samples is increased, the solution tended toward zero flux as expected. This method gave a quantitative measure of sampling errors, and guided the choice of the necessary number of rays. In cases where very large numbers of rays could not be afforded, a correction could be applied. The radiative flux error from the preprocessor step can be added to all later simulated net radiative fluxes to correct for sampling errors in emission calculations.

\subsection{Energy balance equation}

To determine leaf surface temperature, separate energy conservation equations are solved for either individual or volumes of leaves. Although not a focus of this paper, the modeling system also requires realistic specification of ground surface temperature. There is an extensive body of previous literature focused on developing complex land surface energy balance models (e.g., Noilhan and Planton, 1989; Ács et al., 1991; Chen et al., 1996). Instead, we used a simple model for ground temperature, which incorporates measurements to calculate some of the unknown terms. For completeness, the procedure for calculating ground surface temperature is detailed in Appendix A.

\subsubsection{Energy balance of an individual leaf}

The energy transport model is based on the underlying strategy of aggregating individual leaves into homogeneous discrete volumes and ultimately solving for the integrated surface temperature (detailed below in Sect. 2.3.2). However, to compare model predictions with point measurements of leaf temperature, individual leaves were also inserted into the simulated domain. Individual leaf surface temperature was determined by solving the familiar leaf energy balance equation (where we assume net radiation balances sensible and latent heat 
fluxes), which can be written as (Campbell and Norman, 1998)

$$
\frac{R_{L}}{2 A_{L}}-\varepsilon_{L} \sigma T_{L}^{4}=c_{p} g_{H}\left(T_{L}-T_{a}\right)+\frac{1}{n} \lambda g_{M} \frac{\left(e_{s}\left(T_{L}\right)-e_{a}\right)}{p_{a t m}},
$$

where $R_{L}$ is the net incoming radiation energy rate absorbed at the leaf surface (see Appendix B), $A_{L}$ is the one-sided leaf surface area, $\varepsilon_{L}$ is the leaf emissivity, $\sigma=5.67 \times 10^{-8} \mathrm{~W} \mathrm{~m}^{-2} \mathrm{~K}^{-4}$ is the Stefan-Boltzmann constant, $c_{p}$ is the specific heat capacity of air, $g_{H}$ is the leaf boundary layer conductance to sensible heat, $T_{L}$ and $T_{a}$ are leaf and air temperature, respectively, $n=1$ for hypostomatous leaves or $=2$ for amphistomatous leaves, $\lambda$ is the latent heat of vaporization for water, $g_{M}$ is the leaf conductance to moisture, $e_{s}\left(T_{L}\right)$ is the saturation vapor pressure evaluated at the leaf surface temperature, $e_{a}$ is the air vapor pressure, and $p_{a t m}$ is the atmospheric pressure.

The boundary layer conductance to heat is approximated by assuming the leaf is an isothermal inclined flat plate in laminar mixed free/forced convection. The orientation of the leaf is given in spherical coordinates, with $\theta_{L}$ denoting the leaf inclination angle with respect to horizontal and $\phi_{L}$ denoting the azimuthal angle of the leaf normal. We used the correlations of Chen et al. (1986) to write $g_{H}$ for leaf inclination angles of $\theta_{L}<75^{\circ}$ as

$$
g_{H}\left(\theta_{L}\right)=\frac{\rho_{a} \nu}{\operatorname{Pr} D_{L}} 2 F_{1} \operatorname{Re}^{1 / 2}\left\{1 \pm\left[\frac{2 F_{2}\left(G r \cos \theta_{L} / \operatorname{Re}^{2}\right)^{1 / 4}}{3 F_{1}}\right]^{n}\right\}^{1 / n},
$$

and for $\theta_{L}>75^{\circ}$ as

$$
g_{H}\left(\theta_{L}\right)=\frac{\rho_{a} \nu}{\operatorname{Pr} D_{L}} 2 F_{1} \operatorname{Re}^{1 / 2}\left\{1 \pm\left[\frac{F_{3}\left(G r / R e^{5 / 2}\right)^{1 / 5} G r C\left(\theta_{L}\right)}{6\left[0.2+C\left(\theta_{L}\right)\right] F_{1}}\right]^{n}\right\}^{1 / n}
$$

where $\rho_{a}, \nu$, and $\operatorname{Pr}$ are respectively the molar density, kinematic viscosity, and Prandtl number of air, $D_{L}$ is the effective leaf dimension (cf. Parkhurst et al., 1968), Re is the Reynolds number based on $D_{L}$ local free-stream air velocity, and $G r$ is the Grashof number which is defined here as

$$
G r=\frac{g \beta\left(T_{L}-T_{a}\right) D_{L}^{3}}{\nu^{2}},
$$

where $g$ is the acceleration due to gravity, and $\beta$ is the volumetric thermal expansion coefficient which we approximate as $1 / T_{a}$ with $T_{a}$ in units of Kelvin. The plus and minus signs in Eqs. 2 and 3 correspond to buoyancy assisting flow and opposing flow cases, respectively. In the present model, the mean wind vector is always orthogonal to the gravity vector (transverse flow), and thus 
we always take the positive or buoyancy assisting flow case. The constants in Eqns. 2 and 3 are defined as

$$
\begin{aligned}
& F_{1}=0.399 \operatorname{Pr}^{1 / 3}\left[1+(0.0468 / \operatorname{Pr})^{2 / 3}\right]^{-1 / 4}, \\
& F_{2}=0.75 \operatorname{Pr}^{1 / 2}\left[2.5\left(1+2 \operatorname{Pr}^{1 / 2}+2 \operatorname{Pr}\right)\right]^{-1 / 4}, \\
& F_{3}=\operatorname{Pr}^{1 / 2}\left[0.25+1.6 \operatorname{Pr}^{1 / 2}\right]^{-1}(\operatorname{Pr} / 5)^{0.2+C\left(\theta_{L}\right)}, \\
& C\left(\theta_{L}\right)=0.070\left(\cos \theta_{L}\right)^{1 / 2} .
\end{aligned}
$$

We note that effects of leaf azimuth $\phi_{L}$ on $g_{H}$ were neglected. Chen et al. (1986) mention that Eq. 2 is valid for $10^{3} \leq R e \leq 10^{5}$, and Eq. 3 is valid for $10^{3} \leq G r P r \leq 10^{9}$. We expect leaf Reynolds numbers somewhere between $5 \times 10^{3}$ and $5 \times 10^{4}$. Average $G r P r$ values are usually on the order of $10^{6}$. When $T_{L} \approx T_{a}$, it is possible for $\operatorname{Gr} \operatorname{Pr}$ to drop below $10^{3}$, however in these cases the net radiation is nearly zero and convection is not important.

Stomatal conductance, $g_{s}$, is required for the calculation of $g_{M}$. It was calculated using the model proposed by Buckley et al. (2003) and Buckley et al. (2012), with some notable improvements. The base equation can be written as

$$
g_{s}=\frac{\left(\psi_{s}+\pi_{e}\right)\left(i+i_{0}\right)}{k+b i+R\left(i+i_{0}\right) D},
$$

where $i$ is the PAR photon flux, $D=\left[e_{s}\left(T_{L}\right)-e_{a}\right] / p_{a t m}$ is the vapor pressure saturation deficit, $\psi_{s}$ is the soil moisture potential, and $R, \pi_{e}, i_{0}, k$, and $b$ are semi-empirical parameters. Although each of the parameters are treated as empirical, they each have physical meaning (Buckley et al., 2003) and could be explicitly calculated. $R$ is the leaf hydraulic resistance, $\pi_{e}$ is the epidermal osmotic pressure, $i_{0}$ is a PAR flux offset that accounts for transpiration in darkness, $1 / k$ is the product of the turgor-to-conductance scaling factor and the initial slope of the response of guard cell advantage to irradiance, and $1 / b$ is the product of the turgor-to-conductance scaling factor and the maximum guard cell advantage. The procedure for estimating these parameters is detailed in Appendix C. Buckley et al. (2012) lumped $\left(\psi_{s}+\pi_{e}\right) / R$ into a single parameter that represents the transpiration rate at very high vapor pressure deficit $\left(E_{\max }\right)$, which was assumed constant. This can be problematic, particularly in cases where water availability varies dramatically such as in irrigated plants. Thus, we retained the form that allows $\psi_{s}$ to vary instantaneously. This form comes with the drawback that we must introduce the additional parameter $\pi_{e}$. $\psi_{s}$ was calculated using the empirical relationship of McCumber and Pielke (1981).

Another advantage of our procedure over that of Buckley et al. (2012) is that our radiation model gives a highly accurate and spatially explicit prediction of $i$, rather than using a single above-canopy value. This prevents $g_{s}$ for the entire tree from simultaneously saturating at high irradiance. 
Finally, the overall conductance to moisture can be calculated as

$$
g_{M}=\frac{\left(0.96 g_{H}\right) g_{s}}{\left(0.96 g_{H}\right)+g_{s}}
$$

where we have assumed that the leaf boundary layer conductance to moisture is $0.96 g_{H}$ (Forseth and Norman, 1993).

Equation 1 was solved iteratively for leaf temperature using the NewtonRaphson method until the absolute change in temperature was less than $0.01^{\circ} \mathrm{C}$.

\subsubsection{Energy balance of a leaf volume}

The balance between incoming and outgoing energy from a volume of vegetation is similar to the above equation for an individual leaf, except that we integrate over all leaves in the volume. The budget equation can be written as

$$
\frac{R_{V}}{2 a_{L} V}-2 \varepsilon_{L} \sigma T_{L}^{4} \int_{4 \pi} \frac{f_{V}(\theta, \phi) G(\theta)}{4 \pi} d \Omega=c_{p} \bar{g}_{H}\left(T_{L}-T_{a}\right)+\lambda \frac{\bar{g}_{M}}{n} \frac{\left(e_{s}\left(T_{L}\right)-e_{a}\right)}{p_{a t m}}
$$

where $R_{V}$ is the net radiation energy rate absorbed within the volume (Watts) which is an output of the radiation sub-model (Bailey et al., 2014), $V$ is the volume of the discrete cell of leaves, $a_{L}$ is the one-sided leaf area density of the volume, $f_{V}$ is the fraction of total emitted energy by all leaves in the volume that exits the volume in direction $(\theta, \phi)$ without being re-absorbed (see Bailey et al., 2014), $G(\theta)$ is the fraction of leaf area projected in the direction of $\theta$ (Ross, 1981), $d \Omega$ is a differential solid angle, and $\bar{g}_{H}$ and $\bar{g}_{M}$ are the average conductances to heat and moisture integrated over all leaf normals.

The average boundary layer conductance to heat was calculated by multiplying Eqs. 2 and 3 by the leaf angle probability distribution function and integrating over all possible leaf angles (assuming azimuthal symmetry)

$$
\bar{g}_{H}=\int_{0}^{\pi / 2} g_{H}\left(\theta_{L}\right) g_{L}\left(\theta_{L}\right) \mathrm{d} \theta_{L}
$$

where $g_{L}\left(\theta_{L}\right)$ is the probability that a leaf is oriented such that its normal is in the direction $\theta_{L}$. Note that by definition $\int_{0}^{\pi / 2} g_{L} \mathrm{~d} \theta_{L}=1$. The numerical discretization of $\theta_{L}$ is chosen such that further refinement does not change the value of $\bar{g}_{H}$ (e.g., 50 intervals).

The average conductance to moisture is calculated by replacing $g_{H}$ with $\bar{g}_{H}$ in Eq. 7 and by providing the stomatal conductance model (Eq. 6) with quantities averaged over all leaves in the discrete volume.

Equation 8 can be solved iteratively for leaf surface temperature. However, since the predicted leaf and ground temperatures are coupled throughout the domain, the emission component of the radiation model must also be iterated to convergence. Both the solution of Eq. 8 and the emission exchange were iterated until the absolute change in temperature was less than $0.01^{\circ} \mathrm{C}$. 
Note that we do not explicitly separate the energy balance of sunlit and shaded leaves, as is sometimes necessary when coupling with other models such as photosynthesis. Rather, since $R_{V}\left(V / 2 a_{L}\right)$ is the average radiative flux over all leaf surfaces within the volume, Eq. 8 gives an effective average temperature throughout the volume, which will be useful later during validation. However, Eq. 8 could be easily modified to explicitly calculate sunlit and shaded leaf temperature by replacing $a_{L}$ with the sunlit or shaded leaf area density and separating $R_{V}$ into sunlit or shaded components.

\section{Validation experiment}

Two separate field experiments were designed and conducted to evaluate model performance, which were described in detail by Bailey et al. (2014). The first (VINEYARD) was conducted in a $2 \mathrm{~m}$ tall grapevine canopy (Vitis vinifera) in Oregon, USA $\left(44^{\circ} 49^{\prime} 28^{\prime \prime} \mathrm{N} 123^{\circ} 14^{\prime} 17^{\prime \prime} \mathrm{W}\right)$ between 9 August and 24 August, 2013. Another similar experiment was conducted (IsoTREE) on an isolated $7 \mathrm{~m}$ tall Freeman maple $($ Acer $\times$ Freemanii) on the University of Utah campus ( $40^{\circ} 46^{\prime} 59.9^{\prime \prime} \mathrm{N} 111^{\circ} 50^{\prime} 47.6^{\prime \prime} \mathrm{W}$ ) between 1 July and 31 July, 2013.

For both experiments, various measurements were made in and around vegetation. A $10 \mathrm{~m}$ tall tower was erected to obtain vertical profiles of air-related variables. Local Energy-budget Measurement Stations (LEMS) were positioned at various spatial locations to measure local variables related to the ground surface energy balance (Fernando and Pardyjak, 2013). Details of properties and measurements relating to the radiation sub-model can be found in Bailey et al. (2014). Further measurements and associated equipment relevant to the energy balance model are described below.

\subsection{Leaf temperature measurement}

Traditionally, leaf temperature is difficult to measure in the natural environment (Ziegler-Jöns et al., 1986; Ehleringer, 2000; López et al., 2012). Several methods are available for this task, although each are accompanied by their own trade-offs and uncertainties. In an attempt to gain confidence in leaf temperature measurements and to quantify uncertainty, we used three independent measurement techniques, which are described below.

1. Thermocouples: Thermocouples were attached to individual leaves within the canopy. There are several potential sources of error or limitations when using this measurement method:

- The thermocouple measures temperature at a small point on the leaf. This does not capture variation of temperature across the leaf surface, and thus assumes that the point measurement is representative for the whole leaf. A large number of measurements are required to capture the 3D spatial distribution of temperature. 
- Because of differences in absorptivity/emissivity between the leaf and thermocouple tip, radiation error can occur due to differential heating (Ehleringer, 2000).

- Conduction error can result from heat transfer between the leaf surface and along the thermocouple wire (Tarnopolsky and Seginer, 1999).

- Contact resistance between the thermocouple and the leaf can alter the apparent temperature sensed by the thermocouple tip (Beadle et al., 1973). One option is to insert the thermocouple tip into the leaf tissue, but this causes eventual damage to the surrounding leaf tissue.

We used small diameter thermocouples with low thermal conductivity (40 AWG chromel-constantan; Omega Engineering, Stamford, CT, USA) in an attempt to reduce conduction error (Tarnopolsky and Seginer, 1999). Thermocouples were attached to the lower (abaxial) side of leaves to minimize radiation error, which is quantified in Sect. 3.2. To reduce contact resistance between the thermocouple and the leaf surface, a small amount of OMEGATHERM ${ }^{\circledR}$ thermally conductive paste (Omega Engineering, Stamford, CT, USA) was applied to the thermocouple tip. The insulated portion of the thermocouples were adhered to the leaf using Transpore ${ }^{\mathrm{TM}}$ medical tape (3M, St. Paul, MN, USA), which is permeable to air and moisture.

2. Thermographic imaging: A more complete spatial distribution of leaf temperature was obtained through use of a thermographic camera (FLIR Systems T420, Wilsonville, OR, USA) (Jones, 2004). This gave a twodimensional projection of the leaf temperature distribution (note that this method was only available for IsoTREE). This method can be limited by the following:

- Comparisons between the model and thermographic images are mostly qualitative. When photographing the entire tree it is difficult to pinpoint individual leaves, and since the tree is not an opaque object it is often difficult to separate the tree from the background.

- Since the camera measures radiometric flux, results are limited by knowledge of the surface emissivity as it is a necessary parameter to backcalculate temperature. Furthermore, emissivity can vary within a single image making it impossible to simultaneously quantify the temperature in the entire image (López et al., 2012).

- This measurement method inherently assumes that objects are diffuse (isotropic) emitters. However, the radiation emitted from the tree is directionally-dependent. Thus, if most of the leaves in a given space are not projected normal to the camera, this can result in under prediction of leaf temperature (Hellebrand et al., 2001).

- Unless the camera is equipped with an internal cooling system, measurements will be affected by the body temperature of the camera. Cameras with this feature often come with an order of magnitude increase in price. 
- Interactions with surroundings can alter the perceived surface temperature. Radiation emitted by surrounding objects that is reflected by the object of interest can increase the radiative flux sensed by the camera. Attenuation by the air can also cause difference between the radiative flux at the object and at the camera.

Many of the above issues are difficult to deal with. Image processing (e.g., Jones, 2004) and corrective functions (e.g., Jones et al., 2002) can be used to cancel these effects, but they also introduce their own errors which can be difficult to quantify. We used the camera's software to correct for background radiation and air attenuation errors, although we expect these errors to be small because the emissivity of the leaves is high and the camera was only about 15 meters from the target.

3. Pyrgeometer: The average surface temperature was measured by placing a downward-facing pyrgeometer (radiometer measuring 5,000-50,000 nm wavelengths) above the canopy, for which we used a Kipp \& Zonen CG3 (Delft, Netherlands), which was a component of the Campbell Scientific CNR1 net radiometer. For VINEYARD the pyrgeometer was mounted on a 10-m tall tower, and in IsoTree was placed just above the highest branches.

- The pyrgeometer effectively gives an aggregated radiometric temperature (or emitted radiation flux) of all surrounding objects. Therefore, it gives no specific information on the distribution of temperature.

- The surface emissivity must be known to infer temperature from radiative flux.

\subsection{Uncertainty quantification of leaf temperature measurements}

As detailed above, each of the leaf temperature measurements have (possibly substantial) uncertainty. We used the thermographic camera to assess the uncertainty in the thermocouple point measurements and vice-versa. A large number of thermographic images were taken at $1 \mathrm{~Hz}$ of an individual (thermocoupleequipped) leaf in situ and averaged over a five minute period. The camera was positioned as close to the leaf normal direction as possible. The images were cropped to include only the leaf, and surface temperature was averaged over the whole leaf surface area. The average value was compared to the average thermocouple reading over the same five minute period (Fig. 3).

Agreement between both measurement methods was very good for shaded leaves $\left(\lesssim 1^{\circ} \mathrm{C}\right)$, which suggested that (a) the assumed emissivity of $\varepsilon_{L}=0.97$ was reasonable, and (b) errors due to temperature variation over the leaf surface were minimal (for shaded leaves). For leaves in direct sunlight, the thermocouple consistently reported a higher temperature than the thermographic camera of up to several degrees. This is likely primarily due to radiation error, as solar radiation transmitted through the leaf and absorbed by the thermocouple will raise the measured temperature. We expect that significant errors in the 
thermocouple measurement should be over predictions of leaf surface temperature, and based on Fig. 3 we estimate a maximum thermocouple measurement error during sunlight hours of around $3{ }^{\circ} \mathrm{C}$, and less than $1{ }^{\circ} \mathrm{C}$ during the night. These values are consistent with estimated errors reported in previous literature (Mellor et al., 1964; Ziegler-Jöns et al., 1986).

This exercise provided an estimate of measurement accuracy between the thermocouples and thermographic camera. However these error estimates were not used to apply any correction to measurements.

\subsection{Whole-plant transpiration}

For VINEYARD, whole-plant ET was measured with the eddy-covariance method at a height of 5 meters using an EC150 open-path gas analyzer (Campbell Scientific, Logan, UT). To obtain the moisture flux coming from just the plants, the moisture flux from the ground must be subtracted. The soil moisture flux was inferred using Decagon 5TM probes at depths of 5 and $50 \mathrm{~cm}$, which measure relative soil moisture content $\eta\left(\mathrm{cm}^{3} \mathrm{H}_{2} \mathrm{O} / \mathrm{cm}^{3}\right.$ soil $)$. The soil moisture flux $q_{s}$ was calculated as (McCumber and Pielke, 1981)

$$
q_{s}=\rho_{H 20}\left(K+D \frac{\mathrm{d} \eta}{\mathrm{d} z}\right),
$$

where $\rho_{H 2 O}$ is the density of water, $K$ and $D$ are the soil conductivity and diffusivity for water, which were also calculated following McCumber and Pielke (1981). The empirical soil parameters necessary to calculate $K$ and $D$ were taken from Clapp and Hornberger (1978) for 'silt loam' (this is also discussed in Appendix B). We found that the soil moisture flux was more than an order of magnitude smaller than the moisture flux above the canopy. Thus, it was assumed that the moisture flux at 5-m was equal to the ET flux coming from the plants. This flux was converted to sap flux by dividing by the ratio of sapwood area to ground area, which was about $3.35 \mathrm{~cm}^{2}$ sapwood per $\mathrm{m}^{2}$ ground.

Transpiration rates for IsoTREE integrated over the whole tree were measured using the Granier sap flux technique (Granier, 1987). As with most other sap flux measurement techniques, the Granier method is known to underestimate sap flux (Steppe et al., 2010). However, the fact that VINEYARD used a different measurement method adds confidence to the overall validation exercise.

Three pairs of copper-constantan thermocouples were installed in the tree at a height of approximately $1.5 \mathrm{~m}$. The upper sensor was supplied with 0.2 $\mathrm{W}$, and was separated from the lower (unheated) sensor by a vertical distance of $10 \mathrm{~cm}$. A required parameter for the Granier correlation is the maximum temperature difference between the upper and lower sensor, which corresponds to the minimum sap flow rate. This parameter was calculated as the maximum daily temperature difference averaged over the whole experiment. We did not apply any corrections to account for variability in flow across the sapwood depth (e.g., Pataki et al., 2011), as the sapwood depth was not substantially larger than the probe depth. The sap flux was then calculated from the sap flow rate using the sapwood area, which was estimated to be $75.4 \mathrm{~cm}^{2}$ based on cores obtained using an increment borer. 


\subsection{Air measurements}

In order to focus this study, transport of heat and moisture through the air was driven by data rather than simulated. Coupling to a model for turbulent airborne transport will be a focus of future work. Air temperature and relative humidity were measured using Sensirion SHT15 sensors (Sensirion AG, Switzerland) placed at several locations around the tree and within the vineyard at a height of $2 \mathrm{~m}$. Spatially averaged values were used in model calculations.

Wind speed was measured using CSAT3 sonic anemometers at several heights (Campbell Scientific, Logan, UT). The three-dimensional wind vector was measured at $20 \mathrm{~Hz}$, from which the mean wind velocity magnitude was calculated and averaged over 15 min periods. For VInEYARD these were placed at heights of $0.73,1.37$, and 1.98 meters, and at 1.5, 4.0, and 7.0 meters in IsoTreE. Linear interpolation was used to determine the wind speed at the height of any leaf or vegetation volume.

\section{Simulation inputs}

Observation periods were chosen to match that of Bailey et al. (2014), which produced $15 \mathrm{~min}$ averaged measurements over two complete diurnal cycles. Measurements over these periods are summarized in Figs. 4 and 5. An additional observation period was used for IsOTREE during the period when thermographic images were collected (DOY 212). Relevant model input parameters and measurement locations are given in Table 1 . The leaf angle probability distribution $g_{L}$ was measured using a digital protractor, and is plotted in Bailey et al. (2014).

The VINEYARD domain consisted of 30 rows of vegetation that covered a ground area of $73 \times 73 \mathrm{~m}^{2}$. Each row was discretized into $1 \times 80 \times 8$ sub-volumes, and the ground was discretized into $146 \times 146$ patches. A total of 500 rays were sent from each discrete volume for diffuse radiation exchange calculations (250 rays per patch), and 2025 rays per patch were used for direct radiation exchange calculations.

The IsoTREE domain ground area consisted of a $25 \times 25 \mathrm{~m}^{2}$ area that was discretized into $0.25 \times 0.25 \mathrm{~m}^{2}$ patches. The tree crown was discretized into $10 \times 10 \times 14$ sub-volumes. 750 and 1600 rays were used for diffuse and direct radiative exchange, respectively ( 375 diffuse rays per patch).

The domain discretizations were chosen such that further reducing the size of patches/volumes or increasing the number of rays did not significantly change results. These choices were also guided by the radiative equilibrium approach described in Sect. 2.2.1.

\section{Results}

This section explores the performance of each of the individual components of the energy balance model by comparing to measurements. Agreement between measured values and model prediction were quantified using several measures, 
namely the root mean squared error (RMSE) and index of agreement $d$ (Willmott, 1981). The less robust coefficient of determination $R^{2}$ is also reported, as this is historically the most common measure of agreement in the literature.

\subsection{Whole-plant evapotranspiration}

Figure 6 shows box-and-whisker plots for the modeled vegetation boundarylayer conductance to heat and moisture throughout the experimental period. Each box-and-whisker depicts the variability of the conductance over all the vegetation volumes. Substantial variability across the plants can be observed in conductances for both experimental sites. The boundary-layer conductance to heat showed more spatial variability during the day, although significant variability during the night is also present. The boundary-layer conductance to moisture was highly variable throughout the plants during the day. This is due to extreme variability in solar absorption between sunlit and shaded vegetation. This underscores the importance of representing the three-dimensional variability in complex canopy geometries. At night, there was negligible variability in the boundary-layer conductance to moisture, which results from the fact that the solar radiation flux is negligible.

To assess the performance of the stomatal and boundary-layer conductance sub-models, the model system was used to predict whole-plant ET in terms of sap flux. The simulated sap flux was calculated by summing ET of all discrete volumes over the whole plant according to Eq. C.1. Empirical coefficients required by the stomatal conductance model were determined for VINEYARD using two days worth of data from a different time period (DOY=234-235). Consistent equipment failure in the case of IsoTREE prevented us from obtaining representative model coefficients using separate data. As a result, we used the same data to obtain model coefficients as was used for validation (DOY=190191). For reference, we tried using the same data for obtaining coefficients and validation in VINEYARD and found a minimal impact on results.

Agreement between modeled and measured values was good, with $R^{2}$ above $90 \%$ and $d=98 \%$ for both cases (Fig. 7). Although there were brief periods of significant over or under prediction, most of the model predictions had very low error. On the whole, most model predictions were likely within the error of measurement, although admittedly a sufficient determination of the measurement error was not available.

Inevitably, some of the good model agreement comes from the fact that coefficients were obtained by fitting to data (Appendix C). At the very least, we can say that this test indicates that integrated water transport is consistent within the model. However, more information can also be inferred. First, results suggest that the functional form for $g_{s}$ given by Buckley et al. (2003) is relatively accurate. Additionally, we were able to obtain better agreement between modeled and measured sap flux values using our 3D model instead of the bulk procedure of Buckley et al. (2012) which used only a single measurement of $i$ and $D$ to drive the $g_{s}$ model. Using this bulk procedure, we obtained an $R^{2}$ for IsoTree of 0.88 . This was improved to 0.91 by allowing $\left(\psi_{s}+\pi_{e}\right) / R$ in Eq. 6 to vary. Finally, by using the full 3D model, we obtained the value 
shown in Fig. 7 of 0.92. Minimal improvements were found for VineYARD when incorporating the effects of soil moisture potential and 3D $i$ and $D$, which was likely due in part to the fairly regular canopy geometry as well as the fact that it was not irrigated.

\subsection{Emitted longwave radiation}

Bailey et al. (2014) noted that assuming a constant temperature distribution for plants and the ground likely led to erroneous predictions of longwave emission, especially in the case of isolated vegetation. Here, we attempt to improve predictions of emitted radiation by using a spatially explicit temperature representation rather than using a constant temperature forced by data. In turn, this also serves as validation of the energy balance model's prediction of surface temperature.

Figure 8 shows the present model's predictions of emitted radiation compared against measurements. For VINEYARD, results are similar to that of Bailey et al. (2014) which assumed a constant temperature distribution. This suggests that an average of two point measurements of leaf temperature is sufficient to obtain a representative leaf temperature in VINEYARD. In the case of IsoTREe, results are a marked improvement over the values reported by Bailey et al. (2014). Spatially explicit predictions of 3D surface temperature substantially reduced the under/over prediction of emission flux throughout the day. RMSE decreased from $16.8 \mathrm{~W} \mathrm{~m}^{-2}$ to $7.9 \mathrm{~W} \mathrm{~m}^{-2}$ and $d$ increased from 0.94 to 0.99. Assuming an overall emissivity of 0.96 , we can estimate that this RMSE corresponds to an average overall temperature error of less than $1.5^{\circ} \mathrm{C}$. There was some over prediction in the emission flux in VINEYARD near midday, which was related to an over prediction of ground temperature.

These results indicate that the bulk ground and leaf surface temperature is well represented by the model. Results also emphasize the importance of accounting for the 3D distribution of temperature, even if only bulk abovecanopy values are desired.

\subsection{Individual leaf surface temperatures}

Model predictions for individual leaf surface temperatures were compared against leaf thermocouple measurements (15 min averages) for two complete diurnal cycles (Fig. 9). Figure 10 shows the same data as Fig. 9, but shown as time series rather than scatter plots. Model predictions were excellent throughout the day, with a root mean squared error of less than $2{ }^{\circ} \mathrm{C}$ for both VINEYARD and IsoTree. We estimated in Sect. 3.2 that the average error in thermocouple measurements during daylight was around $2^{\circ} \mathrm{C}$ and likely very low at night, which means that model predictions were probably within measurement error on average. The greatest errors were model under predictions, which usually came at higher temperatures. For VineYARD, the leaves were East-facing and were thus exposed to direct sunlight in the morning. These are the periods in which se see apparent model under prediction. Similarly, IsoTree showed consistent model under prediction when leaves were in direct sunlight. As was 
discussed in Sect. 3.2, this is an expected result and is likely caused by thermocouple radiation error. Thus it is probable that much of the error corresponds to measurement rather than modeling error.

\section{4. $3 D$ leaf surface temperature distributions}

Figure 11 shows box-and-whisker plots of modeled leaf surface temperature throughout the simulation period, which depicts spatial variability in $T_{L}$. In both VinEYARD and IsoTREE, spatial variability is relatively low at night, but is quite large during the day. Both cases show a range in leaf temperatures at midday of around $10^{\circ} \mathrm{C}$ or more. In VINEYARD, sunrise and sunset facilitated very large variance in $T_{L}$ about the mean, whereas at midday variance was small but the range was very large. In IsoTREE, the variance and range were both largest around midday. These results reflect the three-dimensional variability in leaf surface temperature in complex canopy geometries.

To give a qualitative indication of the model's ability to predict the 3D distribution of leaf surface temperature, model visualizations were compared against thermographic images taken during the experimental period (Fig 12). In order to obtain a clearer visualization, individual leaves are plotted although leaf temperature was calculated on a volumetric basis. An individual leaf's surface temperature was visualized by coloring it based on the temperature of the volume in which it resides. Also note that the trunk temperature was not modeled, rather it is simply colored according to a mean leaf temperature for visualization purposes only.

In general, the model is able to capture the 3D temperature distribution within the domain. During the night, the model gives reasonable ground temperature values, which is confirmed by point measurements (see Appendix A). The predicted leaf temperature is very close to values given in the thermographic images and point measurements during nighttime as well as late in the day. In the late morning (10:41), it appears that there may be slight model over prediction, particularly in the shaded area of the tree.

\section{Summary}

A model of the plant canopy energy balance was developed to predict the 3D distribution of energy-related quantities in canopies with complex architectures. Individual plants were discretized into homogeneous volumes, and the ground was discretized into rectangular patches. The model was built on the radiation modeling framework of Bailey et al. (2014), which includes radiation physics commonly neglected in other models such as anisotropic scattering and emission. Complex physics are afforded by performing computations on graphics processing units (GPUs) and utilizing optimized GPU ray-tracing libraries. This allows for the simulation of canopy-scale domains on commodity desktop computers.

The ultimate goal of the present model was to accurately and efficiently predict the $3 \mathrm{D}$ distribution of leaf surface temperature by solving the energy 
balance for discrete volumes of leaves. A new approach was used to calculate the radiative emission term in the energy balance which realistically accounts for emission and re-absorption within the volume. Boundary-layer conductances to heat and moisture were calculated using a novel approach, which incorporates the full distribution of leaf angles within the volume.

As a simple model test, we verified that the model adhered to the second law of thermodynamics, or that for a homogeneous temperature distribution the model predicts a zero net flux at every surface. This also guided decisions regarding choices on the necessary discretization level. We found that not using enough ray samples relative to the size of the spatial grid could lead to undersampling errors that cause the model to violate the second law of thermodynamics. To validate model predictions, a set of experiments were designed to simultaneously measure model outputs at many different spatial locations. Two separate experiments were performed: one in a grapevine canopy (VINEYARD) and another in and around a relatively isolated urban tree (ISOTREE). Since leaf surface temperature is notoriously difficult to measure in the natural environment, three independent methods were used. This increased confidence in measured values and allowed for the estimation of measurement errors.

Correctly representing the 3D spatial distribution of temperature substantially improved predictions of above-canopy fluxes of emitted radiation compared to an assumption of spatial temperature homogeneity. This result emphasized the need to account for the spatial distribution of temperature, even when only above-canopy bulk values are of interest.

Predictions of individual leaf temperatures proved to be highly accurate, with daily root mean square errors below $2^{\circ} \mathrm{C}$. This was roughly within the estimated error of the point measurements of leaf surface temperature. Model predictions of 3D surface temperature were assessed by comparing with thermographic images of IsoTreE. The model qualitatively represented the surface temperature distribution throughout a diurnal cycle.

Based on the results of the performed validation tests, we feel the model is well-suited to not only drive other biophysical models, but also to study physical processes in complex canopy environments. This could involve scaling leaf-level processes to whole canopies and studying interactions between various scales without assumptions of canopy-scale homogeneity (Ehleringer and Field, 1993). The model is also ideal for application to urban canopy problems where a wide range of transport scales are important (e.g., Addepalli et al., 2013; Overby et al., 2016). Examining various outputs of the model and how they behave physically is a planned direction for future work.

\section{Acknowledgments}

The authors are indebted to J. Johnson, the laboratory of W. Mahaffee, as well as numerous others for their support with the field experiments. This research was supported by National Science Foundation grants IDR CBETPDM 1134580, EPS 1208732, and AGS 1255662. Any opinions, findings, and 
conclusions or recommendations expressed are those of the authors and do not necessarily reflect the views of the National Science Foundation.

\section{Appendix A. Ground energy balance}

An energy balance can be written at the interface between the soil and atmosphere. A schematic depicting this balance is given in Fig. 13. The model equation for ground temperature can be expressed as

$$
R_{N}-\varepsilon_{G} \sigma T_{G}^{4}-Q_{G}=c_{p} g_{H}\left(T_{G}-T_{a}\right)+\lambda g_{M} \frac{e_{s}\left(T_{G}\right)-e_{a}}{p_{a t m}} .
$$

$R_{N}$ is the net absorbed radiation flux, which is calculated from the radiation transport model. $\varepsilon_{G} \sigma T_{G}^{4}$ is the flux due to radiative emission. $g_{H}$ is the conductance to convection heat transfer, which was assumed spatially constant. It was calculated as $g_{H}=Q_{H}(z=5 \mathrm{~m}) /\left[c_{p}\left(T_{G}-T_{a}\right)\right]$ where the sensible heat flux at $5 \mathrm{~m} Q_{H}(z=5 \mathrm{~m}), T_{a}$, and $T_{G}$ were measured at the eddy covariance station. Although this methodology ignores contributions from the plants to $g_{H}$, it was used as a rough estimation with the hope of obtaining a reasonable ground temperature prediction. The ground temperature was measured using infrared thermometers: in VINEYARD using model SI-111 (Apogee Instruments, Logan, UT, USA) and in IsoTreE using model TN9 (ZyTemp, Hsin Chu, Taiwan). The conductance to moisture transfer $g_{M}$ was also calculated from the latent heat flux $Q_{E}$ at the eddy covariance station as $g_{M}=Q_{E}(z=5 \mathrm{~m}) /\left[\lambda\left(e_{s}\left(T_{G}\right)-e_{a}\right) / p_{a t m}\right]$. As with $g_{H}$, this procedure for estimating $g_{M}$ at the ground is not physically correct. Since it includes the combined effect of evaporation from the ground and plants, predicted values of $g_{M}$ are probably too large. However, this methodology gave values that were adequate for reasonable prediction of ground temperature. Note also that we assumed evaporation occurred at full potential (i.e., saturated) and that $g_{M}$ captured any effects of non-saturation. $Q_{G}$ is the energy flux into the soil, which was calculated using the soil temperature measurements at two depths and the predicted ground surface temperature. Since the temperature gradient at the surface needed to calculate $Q_{G}$ could not be resolved, the heat flux at $z=z_{1}$ was calculated using a finite difference scheme with a three-point, unequally spaced stencil which was extrapolated to the ground surface $(z=0)$ using a storage term. This can be expressed as

$$
Q_{G}=\Delta Q_{s}+K_{H}\left[\frac{\left(z_{2}-z_{1}\right)^{2} T_{G}+\left(z_{1}^{2}-\left(z_{2}-z_{1}\right)^{2}\right) T_{s}\left(z_{1}\right)-z_{1}^{2} T_{s}\left(z_{2}\right)}{\left(z_{2}-z_{1}\right) z_{1} z_{2}}\right]
$$

where $T_{s}(z)$ is the measured soil temperature at absolute depths $z_{1}$ and $z_{2}$ $\left(z_{2}>z_{1}\right) . K_{H}$ is the soil thermal conductivity, which was calculated following McCumber and Pielke (1981), which gives $K_{H}$ as a function of measured soil moisture and four semi-empirical hydraulic parameters. The soil 
was found to be 'silt loam' at both sites according to the USDA soil survey (http://websoilsurvey.nrcs.usda.gov), and thus hydraulic parameters were taken directly from Clapp and Hornberger (1978). For VinEYARD, $K_{H}$ was typically between 0.35 and $0.4 \mathrm{~W} \mathrm{~m}^{-2}{ }^{\circ} \mathrm{C}^{-1}$, and between 1.3 and $1.4 \mathrm{~W} \mathrm{~m}^{-2}{ }^{\circ} \mathrm{C}^{-1}$ for IsoTree. Finally, $\Delta Q_{s}$ is the change in soil heat flux between the ground surface and $z_{1}$ due to storage, which was calculated as

$$
\Delta Q_{s}=C_{v} z_{1} \frac{T_{*}(t)-T_{*}(t-\Delta t)}{\Delta t},
$$

where $T_{*}$ is the average temperature in the layer between $z=0$ and $z=z_{1}$. Since this was not known, it was approximated as $\frac{1}{2}\left(T_{G}+T_{s}\left(z_{1}\right)\right)$. $C_{v}$ is the volumetric heat capacity of the soil, which was calculated (neglecting contributions of air) as (Moene and van Dam, 2014)

$$
C_{v}=\left(1-\eta_{s}\right) C_{p}+\eta C_{w}
$$

where $C_{p}$ and $C_{w}$ are respectively the volumetric heat capacity of soil particulates and water, and $\eta_{s}$ is the saturated value of the relative soil moisture content $(\eta)$. We assumed $C_{p}=1.4 \times 10^{6} \mathrm{~J} \mathrm{~m}^{-3} \mathrm{~K}^{-1}$ (Moene and van Dam, 2014), and $\eta_{s}$ was taken from (Clapp and Hornberger, 1978).

For reference, a comparison of measured and simulated ground surface temperatures is given in Fig. 14. The model reasonably replicates the diurnal trend of ground temperature, with average errors near $2.5^{\circ} \mathrm{C}$.

\section{Appendix B. Radiation calculations for individual leaves}

The net radiation flux on individual leaves was calculated by inserting a leaf 'sensor' into the simulated domain. The sensor does not interact with volumes of vegetation within the domain, as it does not emit or absorb any ray energy. Rather, when a ray passes through the sensor it continues on, but the theoretical absorption is calculated.

When a ray with some energy $E_{\text {ray }}[\mathrm{W}]$ intersects a leaf sensor, some fraction is absorbed $\alpha$, while the rest is transmitted or reflected due to a combination of diffuse and specular reflection. Some fraction of $E_{\text {ray }}$ is specularly reflected by leaf epicuticular wax $\left(\rho_{S}\right)$, leaving $E_{\text {ray }}\left(1-\rho_{S}\right)$ to enter the leaf tissue.

The diffuse absorptivity $\alpha_{D}$ due to absorption by the leaf tissue is not a function of incoming ray direction, and thus it is simply a constant specified based on whether the ray represents the PAR, NIR, or longwave portion of the spectrum (cf. Bailey et al., 2014).

The specular component is calculated using Fresnel's equation

$$
\rho_{S}=\frac{1}{2}\left[\frac{\sin ^{2}\left(\theta_{i}+\theta_{t}\right)}{\sin ^{2}\left(\theta_{i}-\theta_{t}\right)}+\frac{\tan ^{2}\left(\theta_{i}+\theta_{t}\right)}{\tan ^{2}\left(\theta_{i}-\theta_{t}\right)}\right],
$$

where $\rho_{S}$ is the specular reflectance, $\theta_{i}$ is half the angle between the leaf normal and the ray directions (half the vector dot product), $\theta_{t}=\sin ^{-1}\left(\sin \theta_{i} / n\right.$ ) (Snell's 
law), and $n$ is the index of refraction of the leaf wax. The absorptivity $\alpha$ can then be calculated as

$$
\alpha=\left(1-\rho_{S}\right) \alpha_{D} .
$$

The absorbed energy due to all rays intersecting the leaf is summed and divided by the leaf surface area to get the net absorbed radiation flux.

\section{Appendix C. Estimation of semi-empirical stomatal conductance pa- rameters}

As in Buckley et al. (2012), the semi-empirical stomatal conductance parameters in Eq. 6 were calculated using sap flux data. However, a different procedure was used to obtain the spatially explicit distribution of $g_{s}$.

The sap flux can be calculated by summing the stomatal conductance over all discrete volumes in a given plant

$$
f_{s}=\frac{65,448 n}{S_{A}} \sum_{j} g_{M, j}\left(\frac{e_{s}\left(T_{G}\right)-e_{a}}{p_{a t m}}\right)_{j} a_{j} V_{j},
$$

where $f_{s}$ is sap flux i.e., volume of water per sapwood area per time $\left(\mathrm{cm}^{3} / \mathrm{cm}^{2}\right.$ h), $65,448\left(\mathrm{~cm}^{3} \mathrm{H}_{2} \mathrm{O} / \mathrm{mol} \mathrm{H} \mathrm{H}_{2} \mathrm{O}\right) \cdot(\mathrm{sec} / \mathrm{h})$ is a units conversion, $n$ is as defined in Eq. 1, $S_{A}$ is the sapwood area $\left(\mathrm{cm}^{2}\right), a_{j} \cdot V_{j}$ is the one-sided leaf area in the $j^{\text {th }}$ volume. $1 / g_{M, j}=1 / g_{s, j}+1 / 0.96 g_{H, j}$ is the overall conductance to moisture transfer, where $g_{s}$ and $g_{H}$ are calculated locally for each volume $j$ using Eqns. 2,3 , and 6 . The radiation model was run to give the local PAR flux $i$ for an average leaf in each discrete volume.

Since VINEYARD represents an array of plants, the mean sap flux was calculated as

$$
f_{s}=\frac{65,448 n}{r_{s g} A_{G}} \sum_{j} g_{M, j}\left(\frac{e_{s}\left(T_{G}\right)-e_{a}}{p_{a t m}}\right)_{j} a_{j} V_{j},
$$

where $r_{s g}$ is the sapwood area $\left(\mathrm{cm}^{2}\right)$ per unit ground area $\left(\mathrm{m}^{2}\right)$, and $A_{G}$ is the domain ground area. In practice, $A_{G}$ was slightly smaller than the true simulated footprint area to avoid domain edge effects.

Equation C.1 can then be equated to the measured sap flux to give $N$ equations and five unknown parameters $\left(R, \psi_{e}, i_{0}, k\right.$, and $\left.b\right)$, where $N$ is the number of measurement periods. The parameters were found that minimized the error between the measured $f_{s}$ and that calculated from Eq. C.1 or C.2.

\section{References}

Ács, F., Mihailović, D.T., Rajković, B., 1991. A coupled soil moisture and surface temperature prediction model. J. Appl. Meteorol. 30, 812-822. 
Addepalli, B., Pardyjak, E.R., Willemsen, P., Halverson, S.A., Johnson, D.E., Stoll, R., 2013. In search of an intelligent methodology for designing sustainable cities. AWMA EM , 28-32.

Bailey, B.N., Overby, M., Willemsen, P., Pardyjak, E.R., Mahaffee, W.F., Stoll, R., 2014. A scalable plant-resolving radiative transfer model based on optimized GPU ray tracing. Agric. For. Meteorol. 198-199, 192-208.

Beadle, C.L., Stevenson, K.R., Thurtell, G.W., 1973. Leaf temperature measurement and control in a gas-exchange cuvette. Can. J. Plant Sci. 53, 407-412.

Buckley, T.N., Mott, K.A., Farquhar, G.D., 2003. A hydromechanical and biochemical model of stomatal conductance. Plant Cell Environ. 26, 17671785.

Buckley, T.N., Turnbull, T.L., Adams, M.A., 2012. Simple models for stomatal conductance derived from a process model: Cross-validation against sap flux data. Plant Cell Environ. 35, 1647-1662.

Campbell, G.S., Norman, J.M., 1998. An Introduction to Environmental Biophysics. Second ed., Springer-Verlag, New York. 286 pp.

Chen, F., Mitchell, K., Schaake, J., Xue, Y., Pan, H.L., Koren, V., Duan, Q.Y., Ek, M., Betts, A., 1996. Modeling of land surface evaporation by four schemes and comparison with FIFE observations. J. Geophys. Research 101, $7251-7268$.

Chen, T.S., Armaly, B.F., Ramachandran, N., 1986. Correlations for laminar mixed convection flows on vertical, inclined, and horizontal flat plates. J. Heat Trans. 108, 835-840.

Clapp, R.B., Hornberger, G.M., 1978. Empirical equations for some soil hydraulic properties. Water Resources Res. 14, 601-604.

Cook, R.L., 1986. Stochastic sampling in computer graphics. ACM Transactions on Graphics 5, 51-72.

Dai, Y., Dickinson, R.E., Wang, Y.P., 2004. A two-big-leaf model for canopy temperature, photosynthesis, and stomatal conductance. J. Climate 17, 22812299 .

Dauzat, J., Rapidel, B., Berger, A., 2001. Simulation of leaf transpiration and sap flow in virtual plants: Model description and applications to a coffee plantation in Costa Rica. Agric. For. Meteorol. 109, 143-160.

Ehleringer, J.R., 2000. Temperature and energy budgets, in: Pearcy, R.W., Ehleringer, J.R., Mooney, H., Rundel, P.W. (Eds.), Plant Physiological Ecology: Field Methods and Instrumentation. Kluwer Academic Publishers, Dordrecht, The Netherlands, pp. 117-135. 
Ehleringer, J.R., Field, C.B., 1993. Scaling Physiological Processes: Leaf to Globe. Physiological Ecology, Academic Press, Waltham, MA.

Fernando, H.J.S., Pardyjak, E.R., 2013. Field studies delve into the intricacies of mountain weather. Eos, Trans. AGU 94, 313-320.

Forseth, I.N., Norman, J.M., 1993. Modelling of solar irradiance, leaf energy budget and canopy photosynthesis, in: Hall, D.O., Scurlock, J.M.O., BolharNordenkampf, H.R., Leegood, R.C., Long, S.P. (Eds.), Photosynthesis and Production in a Changing Environment: A Field and Laboratory Manual. Chapman \& Hall, London, pp. 207-219.

Granier, A., 1987. Evaluation of transpiration in a Douglas-fir stand by means of sap flow measurements. Tree Physiol. 3, 309-320.

Hellebrand, H.J., Beuche, H., Linke, M., Herold, B., Geyer, M., 2001. Chances and shortcomings of thermal imaging in the evaluation of horticultural products, in: Physical Methods in Agriculture - Approach to Precision and Quality, Prague. pp. 112-117. (ISBN 80-213-0836-2).

Jones, H.G., 2004. Application of thermal imaging and infrared sensing in plant physiology and ecophysiology. Adv. Botan. Res. 41, 107-163.

Jones, H.G., 2014. Plants and Microclimate: A Quantitative Approach to Environmental Plant Physiology. third ed., Cambridge University Press, Cambridge, UK. 407 pp.

Jones, H.G., Stoll, M., Santos, T., de Sousa, C., Chaves, M.M., Grant, O.M., 2002. Use of infrared thermography for monitoring stomatal closure in the field. J. Exp. Botany 53, 2249-2260.

Leuning, R., Kelliher, F.M., DePury, D.G.G., Schulze, E.D., 1995. Leaf nitrogen, photosynthesis, conductance and transpiration: Scaling from leaves to canopies. Plant Cell Environ. 18, 1183-1200.

López, A., Molina-Aiz, F.D., Valera, D.L., na, A.P., 2012. Determining the emissivity of the leaves of nine horticultural crops by means of infrared thermography. Scientia Horticulturae 137, 49-58.

Luquet, D., Begue, A., Vidal, A., Dauzat, J., Clouvel, P., 2003. 3D simulation of directional temperature variability within a row-cotton crop: Toward an improvement of experimental crop water status monitoring using thermal infrared. Precicion Agriculture 4, 297-309.

Mahan, J.R., 2002. Radiation Heat Transfer: A Statistical Approach. John Wiley \& Sons, New York. 482 pp.

McCumber, M.C., Pielke, R.A., 1981. Simulation of the effect of surface fluxes of heat and moisture in a mesoscale numerical model. 1. soil layer. J. Geophys. Res. 86, 9929-9938. 
Mellor, R.S., Salisbury, F.B., Raschke, K., 1964. Leaf temperatures in controlled environments. Planta 61, 56-72.

Moene, A.F., van Dam, J.C., 2014. Transport in the Atmosphere-VegetationSoil Continuum. Cambridge Univ. Press, Cambridge, U. K. 436 pp.

Monteith, J.L., Unsworth, M.H., 2008. Principles of Environmental Physics. third ed., Academic Press, UK. 418 pp.

Noilhan, J., Planton, S., 1989. Parameterization of land surface processes for meteorological models. Mon. Weat. Rev. 117, 536-549.

Overby, M., Willemsen, P., Bailey, B.N., Halverson, S., Pardyjak, E.R., 2016. A rapid and scalable radiation transfer model for complex urban domains. Urban Clim., doi:10.1016/j.uclim.2015.11.004

Parkhurst, D.F., Duncan, P.R., Gates, D.M., Kreith, F., 1968. Wind-tunnel modelling of convection of heat between air and broad leaves of plants. Agric. Meteor. 5, 33-47.

Pataki, D.E., McCarthy, H.R., Litvak, E., Pincetl, S., 2011. Transpiration of urban forests in the Los Angeles metropolitan area. Ecological Applications $21,661-677$.

Ross, J., 1981. The Radiation Regime and Architecture of Plant Stands. Dr. W. Junk Publishers, The Hague, The Netherlands. 424 pp.

Sellers, P.J., Randall, D.A., Collatz, G.J., Berry, J.A., Field, C.B., Dazlich, D.A., Zhang, C., Collelo, G.D., Bounoua, L., 1996. A revised land surface parameterization (SiB2) for atmospheric GCMs. Part I: Model formulation. J. Climate 9, 676-705.

Sinclair, T.R., Murphy, C.E., Knoerr, K.R., 1976. Development and evaluation of simplified models for simulating canopy photosynthesis and transpiration. British Ecol. Soc. 13, 813-829.

Sinoquet, H., Le Roux, X., Adam, B., Ameglio, T., Daudet, F.A., 2001. RATP: A model for simulating the spatial distribution of radiation absorption, transpiration and photosynthesis within canopies: application to an isolated tree crown. Plant Cell Environ. 24, 395-406.

Steppe, K., Pauw, D.J.W.D., Doody, T.M., Teskey, R.O., 2010. A comparison of sap flux density using thermal dissipation, heat pulse velocity and heat field deformation methods. Agric. For. Meteorol. 150, 1046-1056.

Tarnopolsky, M., Seginer, I., 1999. Leaf temperature error from heat conduction along thermocouple wires. Agric. For. Meteorol. 93, 185-194.

Tyree, M.T., Wilmot, T.R., 1990. Errors in the calculation of evaporation and leaf conductance in steady-state porometry: The importance of accurate measurement of leaf temperature. Can. J. For. Res. 20, 1031-1035. 
${ }_{858}$ Wang, Y.P., Jarvis, P.G., 1990. Description and validation of an array model 859 MAESTRO. Agric. For. Meteorol. 51, 257-280.

${ }_{860}$ Willmott, C.J., 1981. On the validation of models. Phys. Geogr. 2, 184-194.

${ }_{861}$ Ziegler-Jöns, A., Knoppik, D., Selinger, H., 1986. The calibration of thermocou${ }_{862}$ ples for leaf temperature measurements in gas exchange cuvettes. Oecologia $863 \quad 68,611-614$. 
Table 1: Model inputs for Vineyard and IsoTree validation test cases. Canopy radiation model inputs are the same as in Bailey et al. (2014). Spatial coordinates are given with $\mathrm{z}=0$ at the ground, and $\{x, y\}=\{0,0\}$ at the middle of the space between the center row in Vineyard and at the tree trunk in IsoTree. $+x$ is North and $+y$ is West.

\begin{tabular}{|c|c|c|c|}
\hline variable & $\begin{array}{l}\text { VINEYARD } \\
\text { value }\end{array}$ & IsoTREE value & units \\
\hline$R$ & $5.33 \times 10^{-4}$ & $1.19 \times 10^{-2}$ & $\mathrm{mmol} \mathrm{m} \mathrm{m}^{-2} \mathrm{~s}^{-1} \mathrm{~Pa}^{-1}$ \\
\hline$\pi_{e}$ & $2.88 \times 10^{6}$ & $1.68 \times 10^{6}$ & $\mathrm{~Pa}$ \\
\hline$i_{0}$ & 112.29 & 3.511 & $\mu \mathrm{mol} \mathrm{m}{ }^{-2} \mathrm{~s}^{-1}$ \\
\hline$k$ & 35404 & 314.04 & $\mu \mathrm{mol} \mathrm{m}{ }^{-2} \mathrm{~s}^{-1} \mathrm{mmol} \mathrm{mol}^{-1}$ \\
\hline$b$ & -25.907 & 11.361 & $\mathrm{mmol} \mathrm{mol}^{-1}$ \\
\hline Leaf \#1 $\{x, y, z\}$ & $\{0,1.1,2.2\}$ & $\{1.73,-1.0,2.6\}$ & $\mathrm{m}$ \\
\hline Leaf $\# 2\{x, y, z\}$ & $\{0,1.05,1.5\}$ & $\{0,0.5,1.8\}$ & $\mathrm{m}$ \\
\hline Leaf \#1 $\left\{\theta_{L}, \phi_{L}\right\}$ & $\{0,0\}$ & $\{0.75,4.7\}$ & $\mathrm{rad}$ \\
\hline 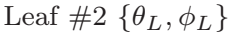 & $\{0.4,4.7\}$ & $\{0.2,1.57\}$ & $\mathrm{rad}$ \\
\hline$D_{L}$ & 0.1 & 0.05 & $\mathrm{~m}$ \\
\hline Patch $\# 1\{x, y, z\}$ & $\{1,0,0\}$ & $\{0.86,2.3,0\}$ & $\mathrm{m}$ \\
\hline Patch $\# 2\{x, y, z\}$ & $\{-1,0,0\}$ & $\{0.79,-2.17,0\}$ & $\mathrm{m}$ \\
\hline
\end{tabular}




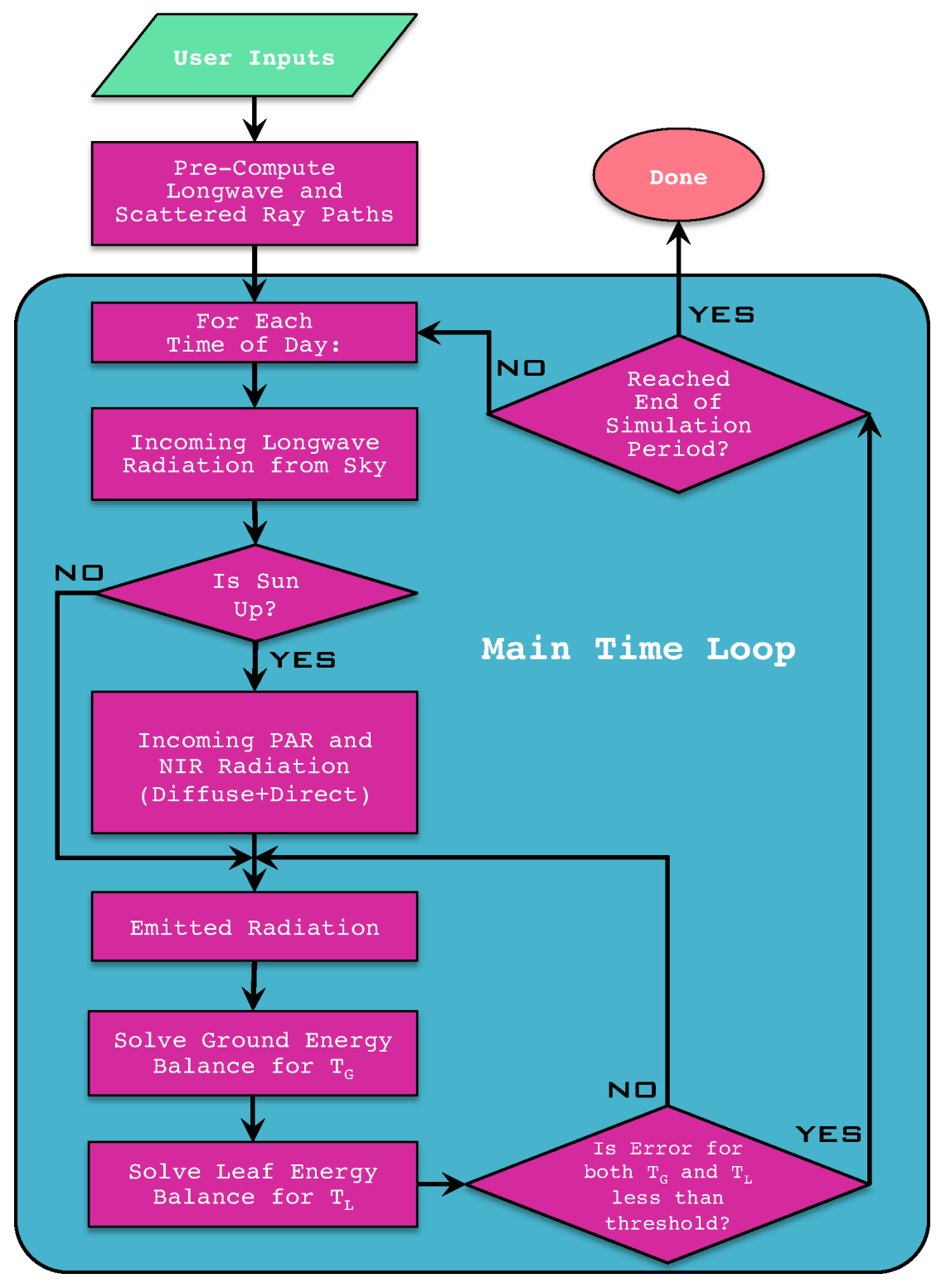

Figure 1: Flowchart depicting the interaction between various sub-models. 


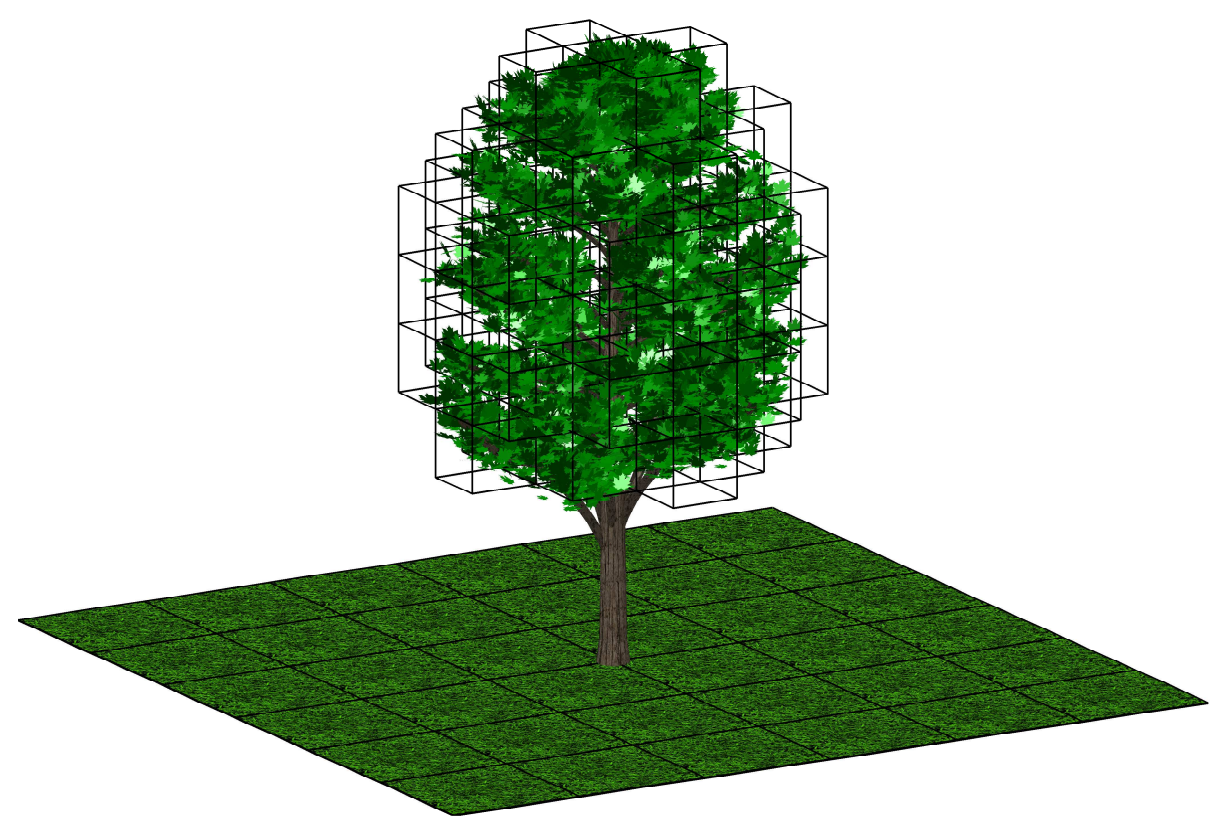

Figure 2: Schematic depicting the way in which the ground surface and vegetation are discretized in the model. The ground is discretized into rectangular sections termed patches. Vegetation is discretized into homogeneous volumes. Reproduced from Bailey et al. (2014) with permission from Elsevier. 


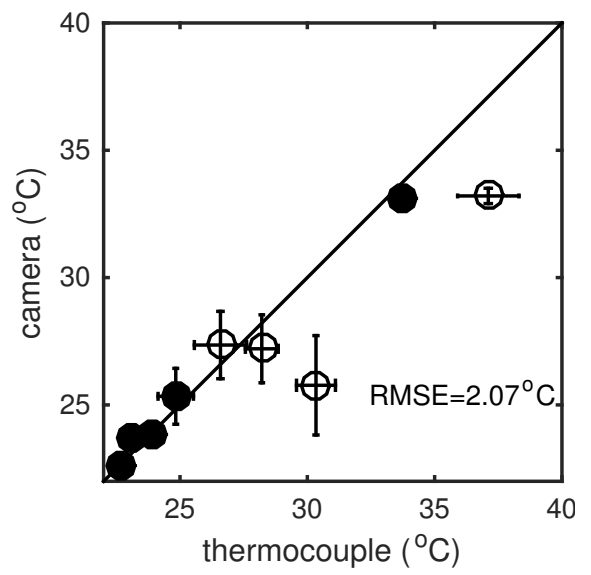

Figure 3: Comparison of measured leaf surface temperature using a thermocouple and a thermographic camera. Horizontal error bars denote the standard deviation of the thermocouple temperature over the temporal averaging period, and vertical error bars denote the standard deviation of the thermographic temperature over the leaf surface. Black and white markers respectively denote shaded and sunlit leaves. 

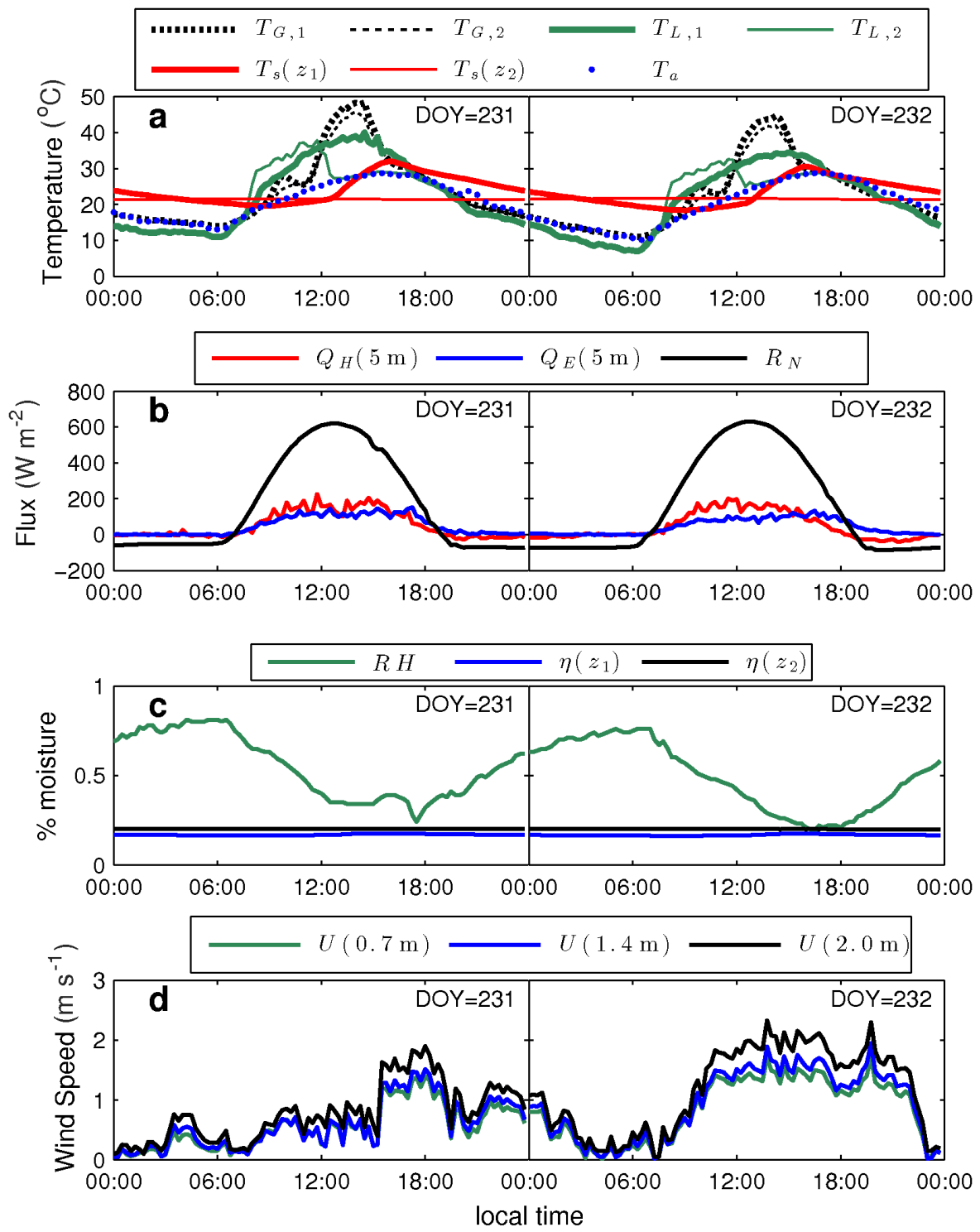

Figure 4: Measurements used to force VINEYARD simulations. (a) ground and leaf surface temperatures $\left(T_{G}\right.$ and $\left.T_{L}\right)$ measured at two spatial locations, and soil temperature measured at two depths $\left(T_{s}\right)$. Air temperature $T_{a}$ is the average value for several locations within the canopy. (b) measured above-canopy fluxes of sensible and latent heat at $5 \mathrm{~m}\left(Q_{H}\right.$ and $\left.Q_{E}\right)$ and net radiation flux. (c) spatially averaged air relative humidity $R H$, and soil water content ( $\eta$ ) measured at two depths $z_{1}=5 \mathrm{~cm}$ and $z_{2}=50 \mathrm{~cm}$. (d) wind speed magnitude at the various measurement heights. 

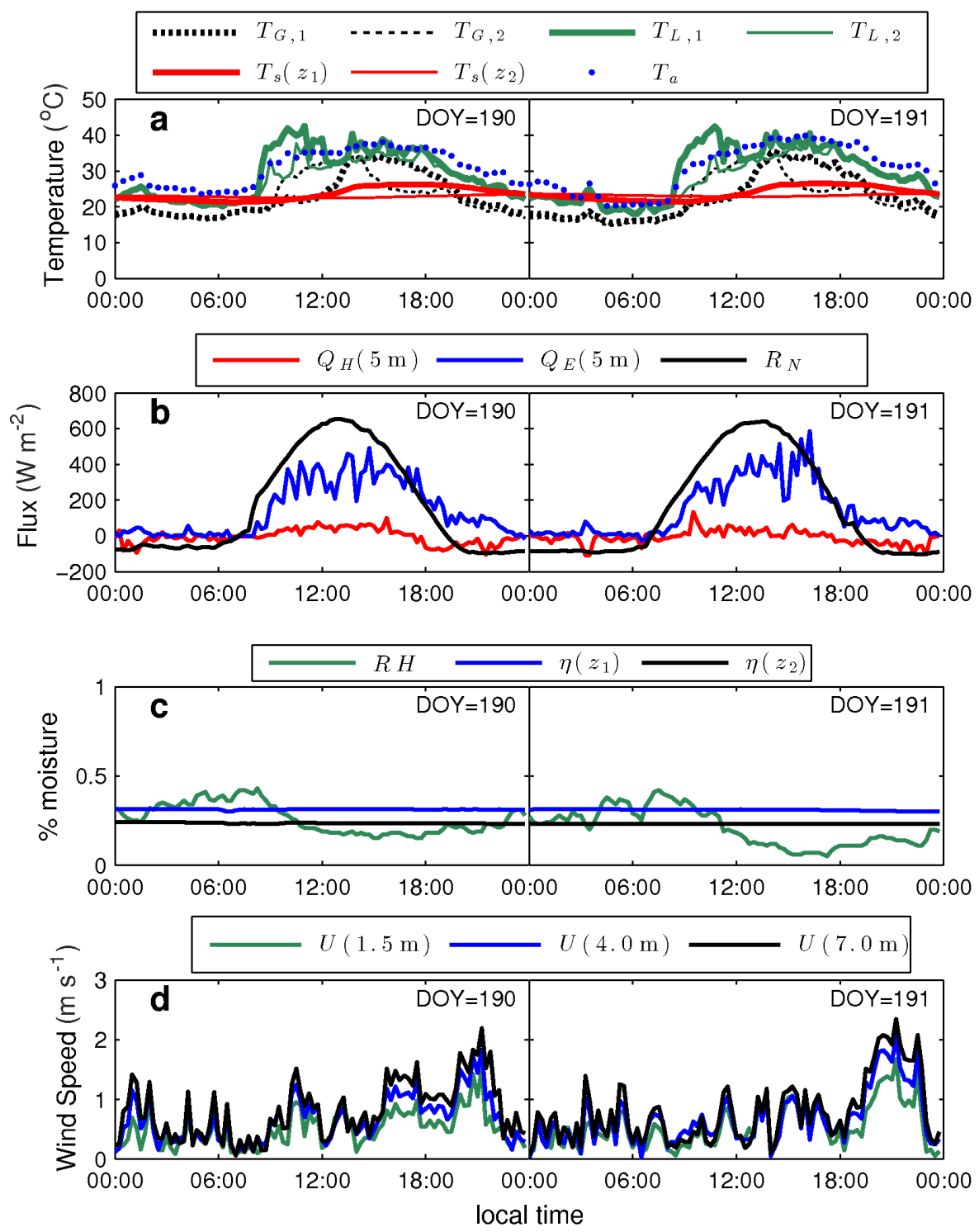

Figure 5: Same as Fig. 4, except for the IsoTreE case. See caption of Fig. 4 for description. 

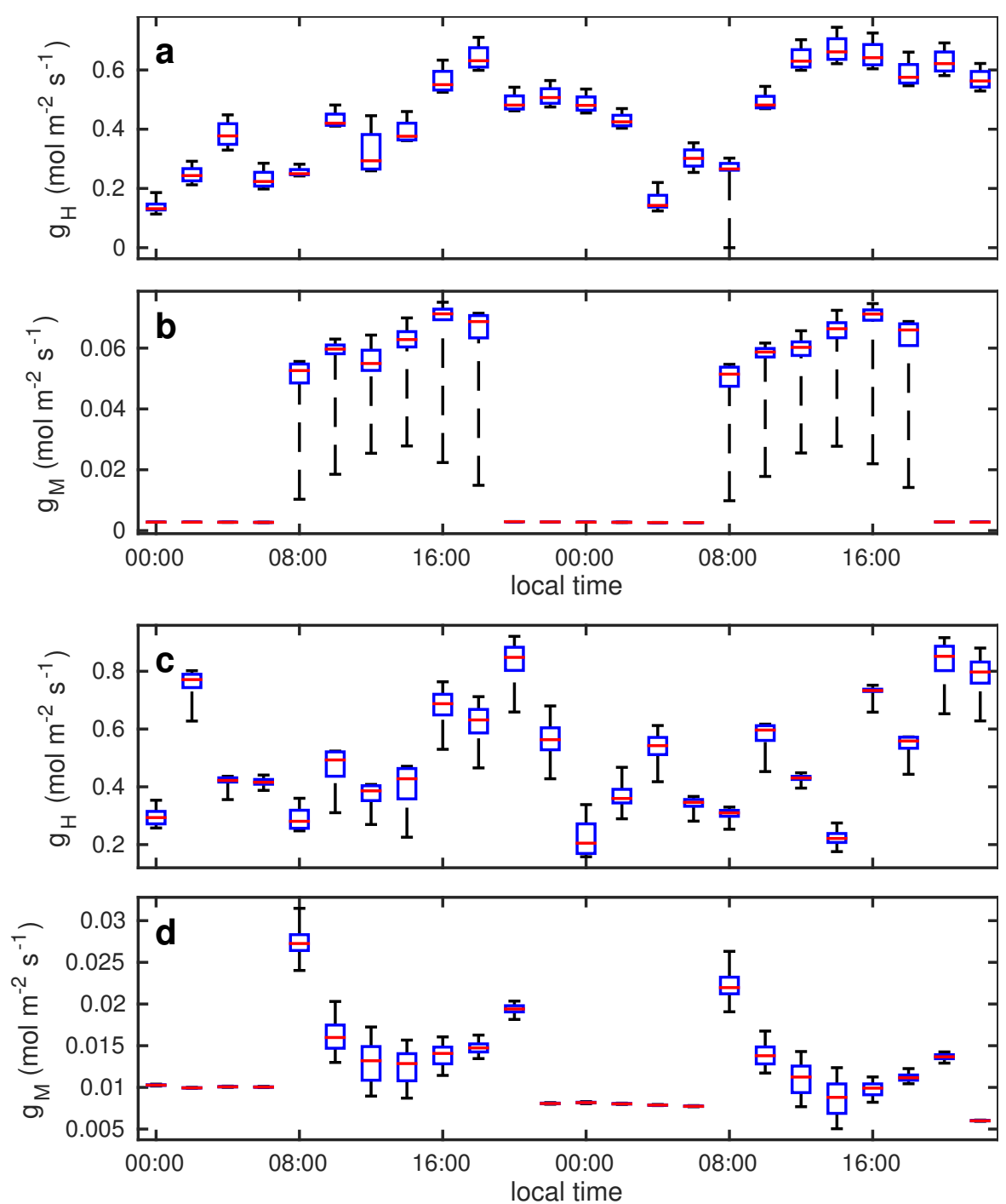

Figure 6: Box-and-whisker plots of the diurnal evolution of modeled vegetation boundary-layer conductance to heat and moisture. Box edges denote the $25^{\text {th }} / 75^{\text {th }}$ percentile, and whiskers denote the full range of values. (a) and (b) correspond to VinEYARD DOY 231-232, (c) and (d) correspond to IsoTREe DOY 191-192. 

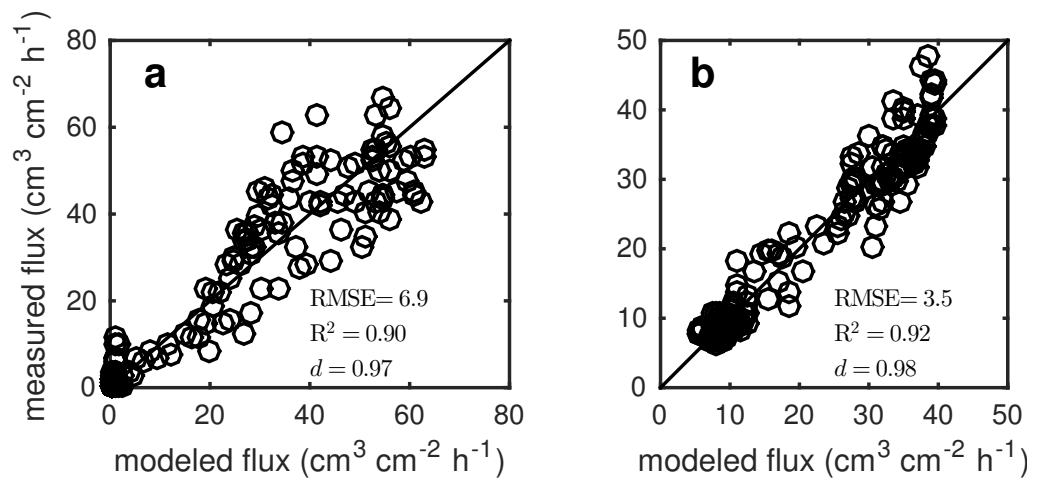

Figure 7: Comparison of measured and modeled values of whole-plant sap flux for (a) VINEYARD and (b) IsOTREE. In (a) empirical coefficients were determined using data from DOY 243-235 (calibration period), and the model predictions given are for DOY 231-232 (validation period). For (b), the calibration and validation periods are both DOY 190-191. 

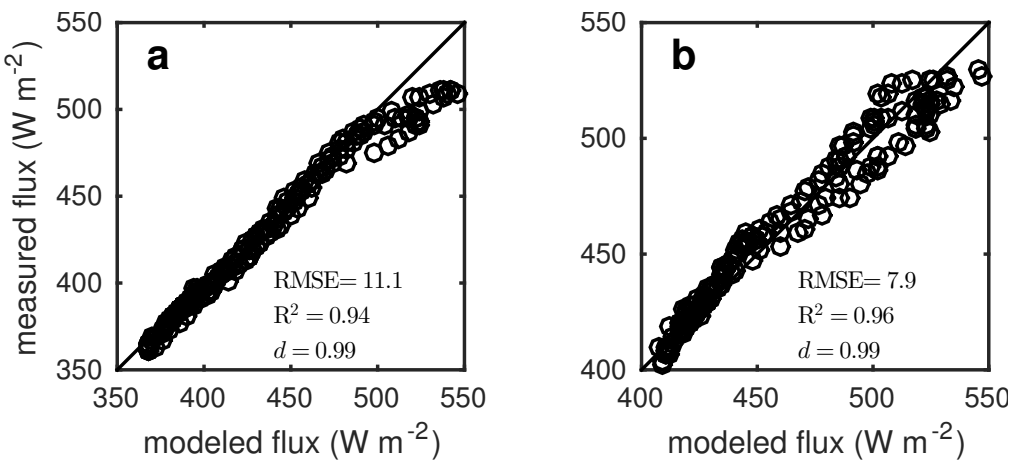

Figure 8: Comparison of measured and modeled values of emitted radiation flux above the canopy for (a) Vineyard and (b) IsoTree. 

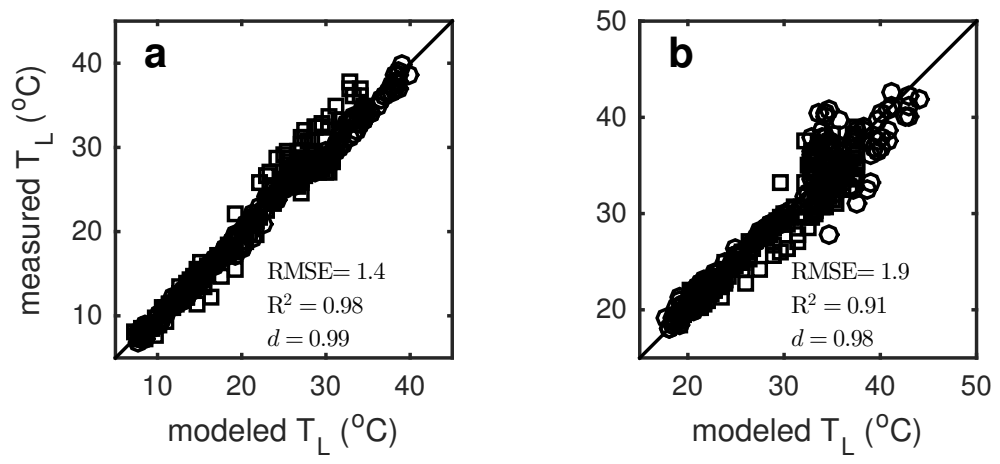

Figure 9: Comparison of measured and modeled values of individual leaf surface temperature for (a) Vineyard and (b) IsoTree. In Vineyard circles correspond to leaves that were near the top of the canopy, and squares correspond to leaves that were near mid-canopy. In IsoTreE circles correspond to leaves that were toward the outside of the tree crown, and were thus often exposed to direct sunlight, and squares correspond to inner leaves that were usually shaded. 

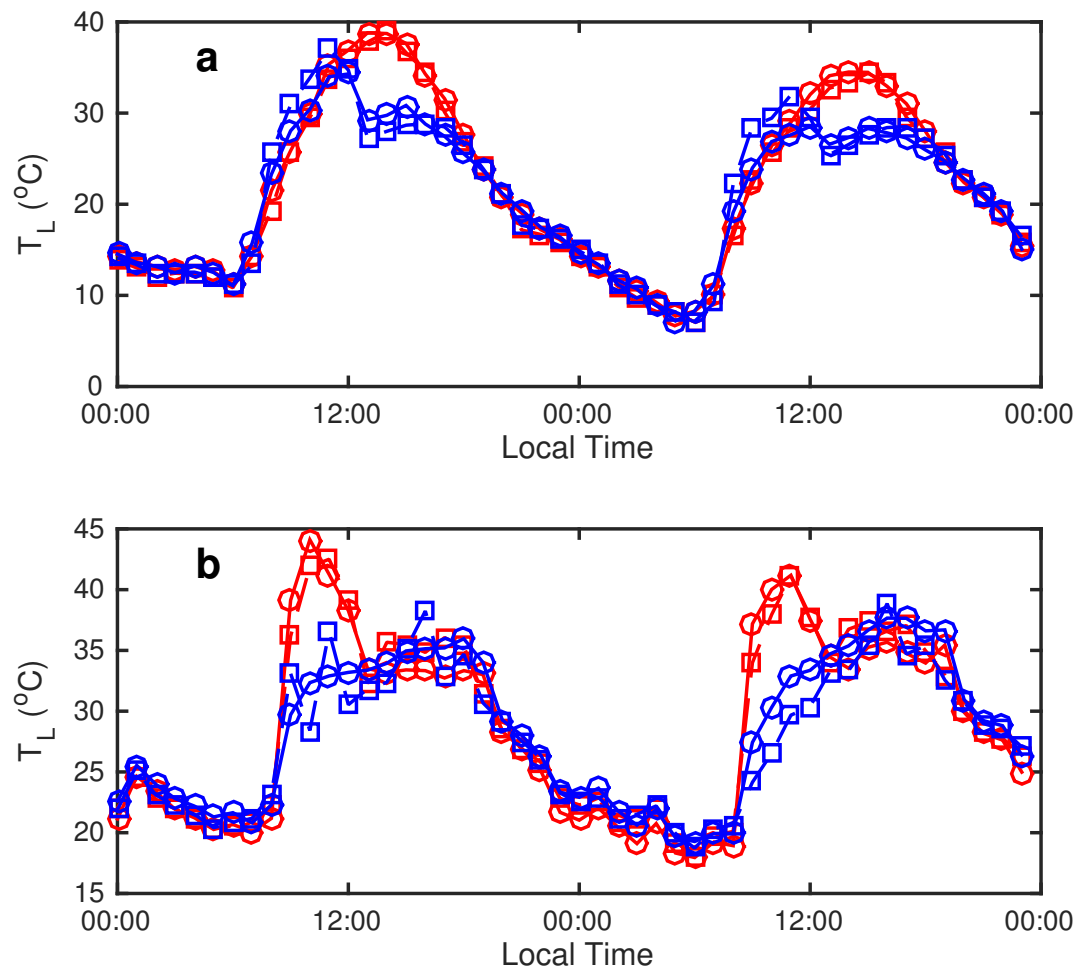

Figure 10: Comparison of measured and modeled values of individual leaf surface temperature for (a) Vineyard and (b) IsoTREe. Squares and circles correspond to modeled and measured values of leaf surface temperature, respectively. Colors denote different measurement locations. For VinEYARD both leaves are East-facing, and blue and red markers denote the leaf at $1.5 \mathrm{~m}$ and $2.2 \mathrm{~m}$, respectively. For IsoTree, blue markers denote the leaf that is West-facing and at a height of $1.8 \mathrm{~m}$, while red markers denote the leaf that is North-West-Facing and at a height of $2.8 \mathrm{~m}$. For visual clarity, only hourly data is shown. 

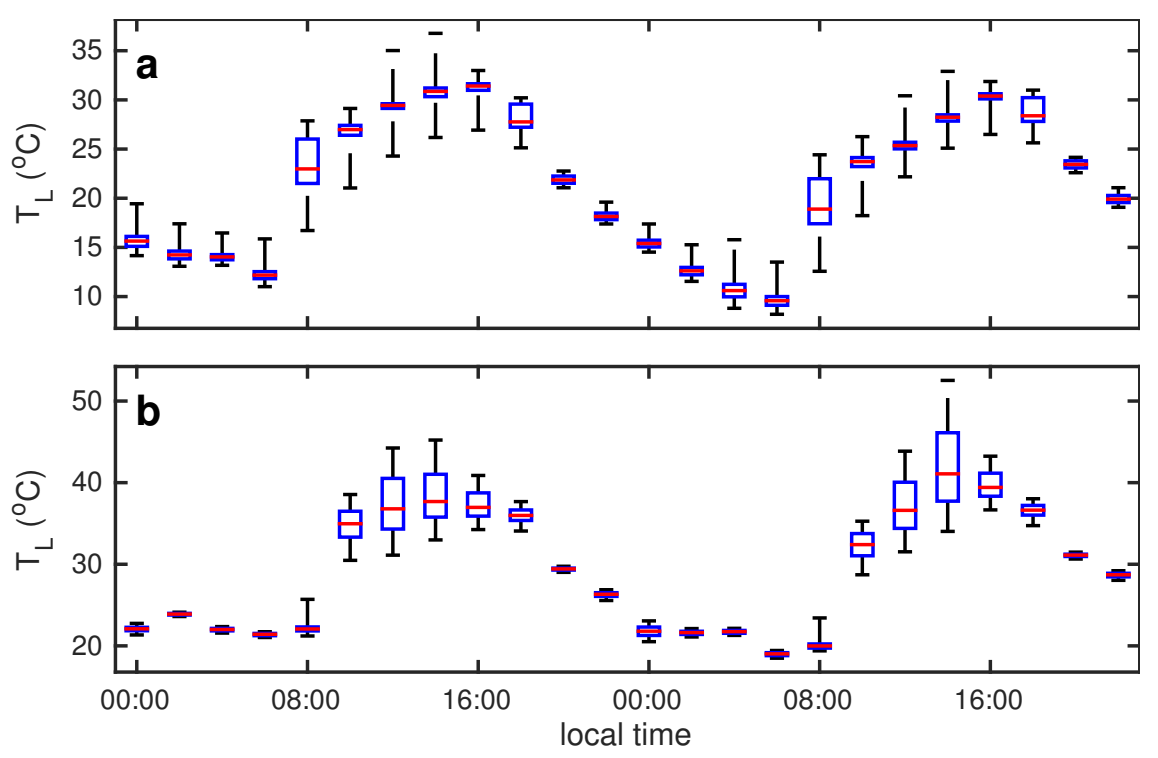

Figure 11: Box-and-whisker plots of the diurnal evolution of modeled leaf temperature. Box edges denote the $25^{\text {th }} / 75^{\text {th }}$ percentile, and whiskers denote the full range of values. (a) and (b) correspond respectively to VineYard DOY 231-232 and IsoTree DOY 191-192. 

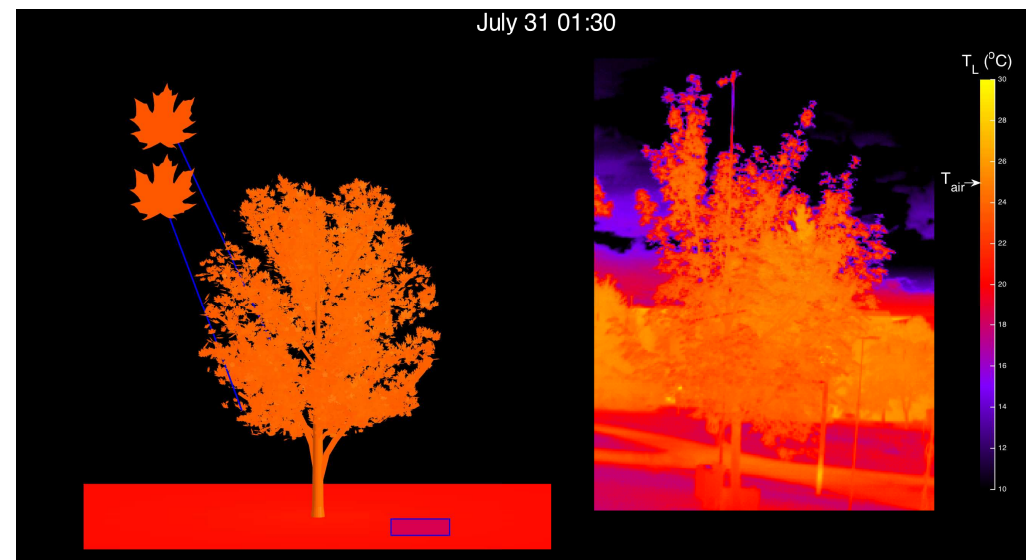

July 31 10:41
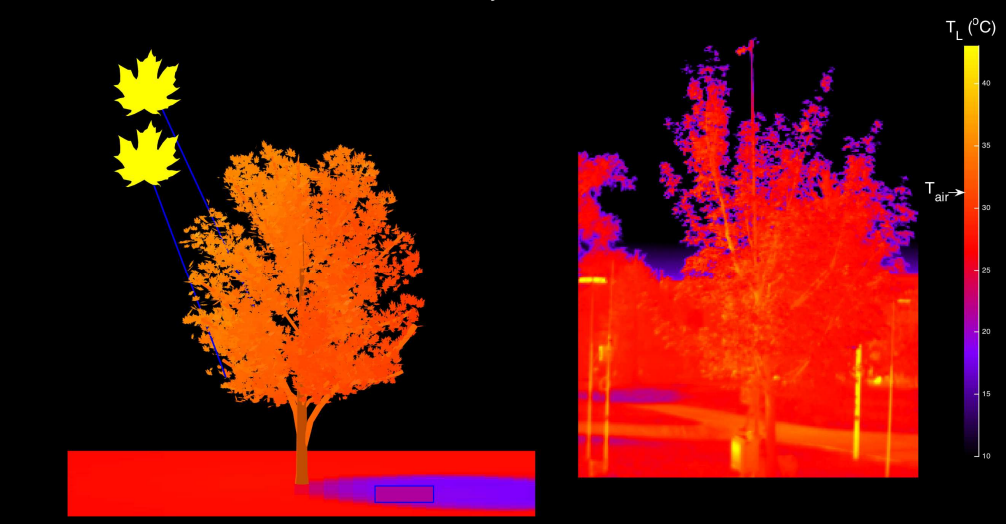

July 31 19:08

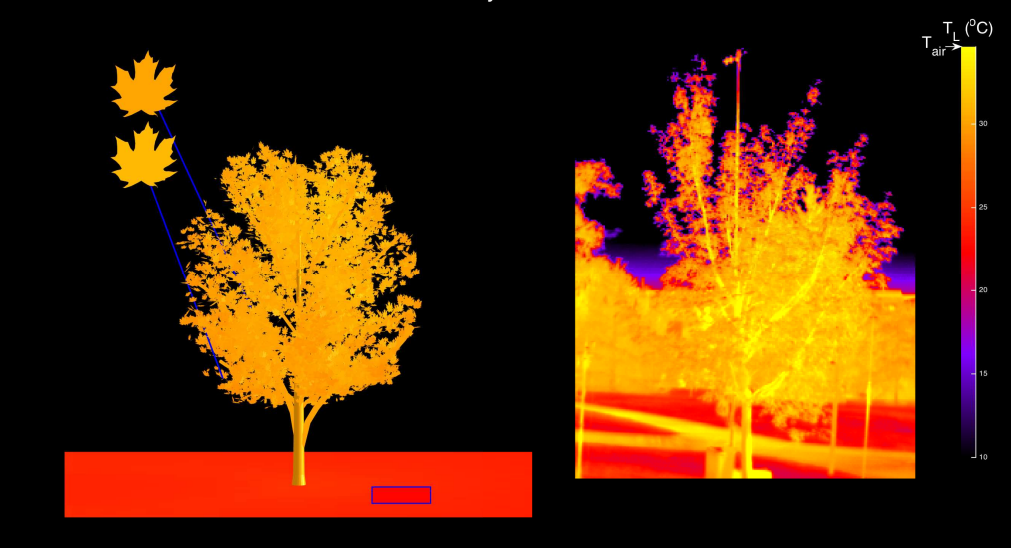

Figure 12: Comparison of simulated (left) and measured (right) 3D surface temperature at three times of day. Simulated leaf temperature is visualized by drawing individual leaves and coloring them based on the temperature of the discrete volume in which they are located. Note that different color scales are used for each image to highlight detail. For reference, point measurements made on two leaves and at one ground location are indicated by blue lines. 


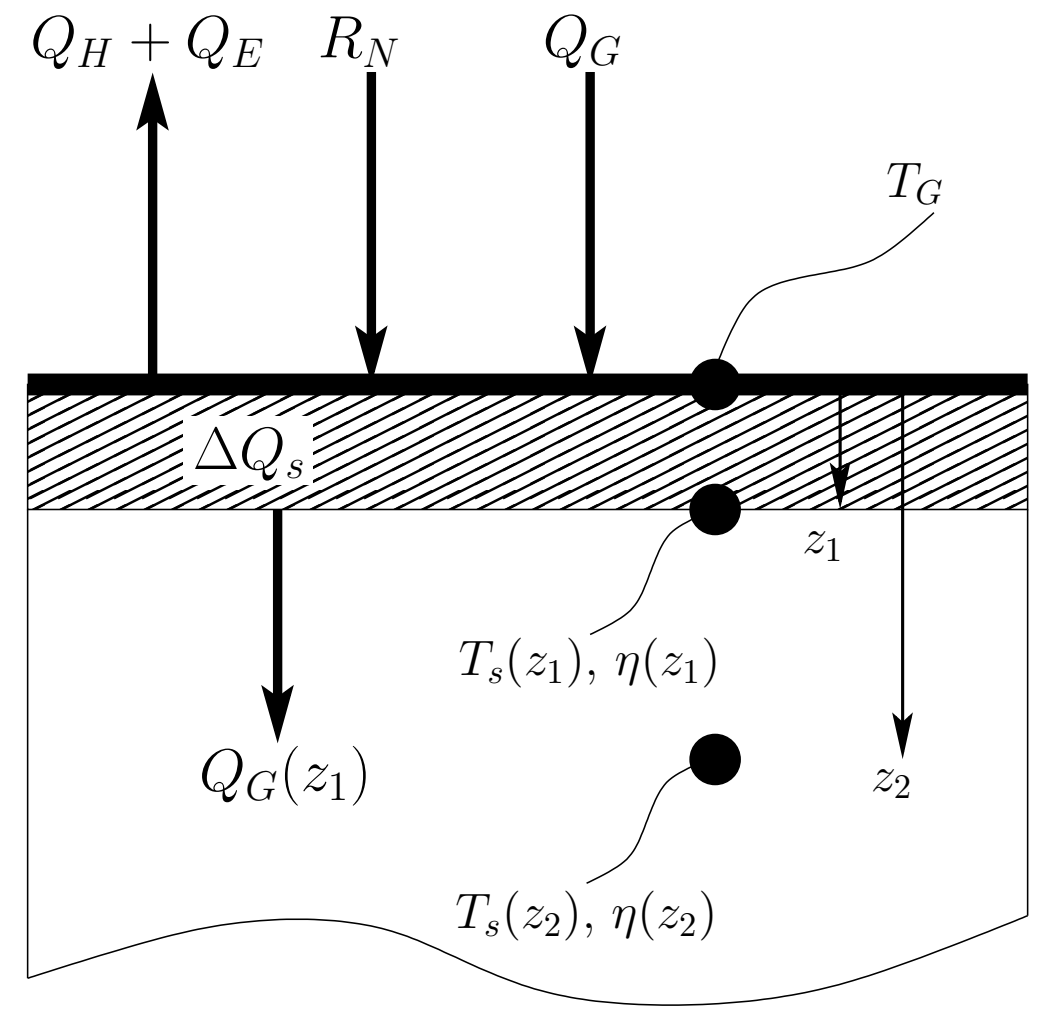

Figure 13: Schematic depicting components of the ground energy balance. The thick horizontal line indicates the energy balance control surface, above which is the atmospheric boundary layer and below which is soil. The hatched area between $z=0$ and $z=z_{1}$ represents the control volume for the calculation of $\Delta Q_{s}$. Filled circles denote measurement locations. 

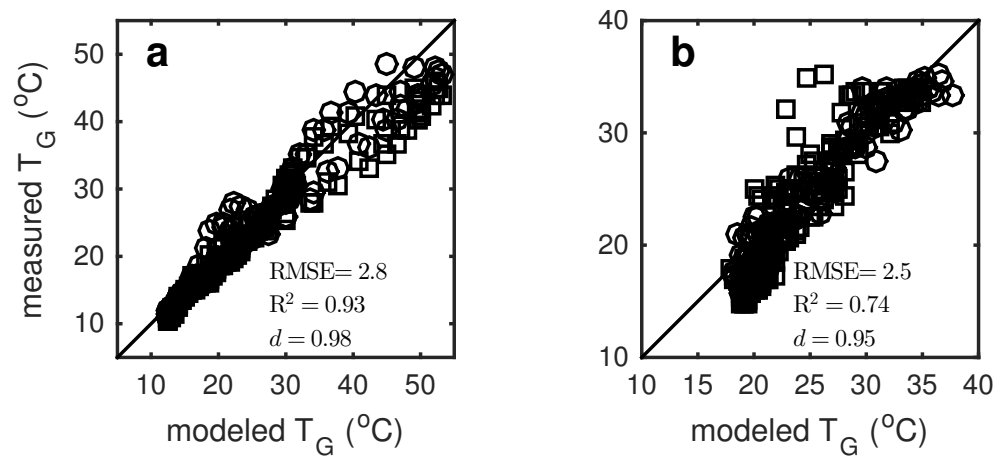

Figure 14: Comparison of measured and modeled values of ground surface temperature for (a) Vineyard and (b) IsoTree. Circles and squares denote different measurement locations. 